\title{
Climatology of estimated LWC and scaling factor for warm clouds using radar - microwave radiometer synergy
}

\author{
Pragya Vishwakarma ${ }^{1}$, Julien Delanoë ${ }^{1}$, Susana Jorquera ${ }^{1}$, Pauline Martinet ${ }^{2}$, Frederic Burnet ${ }^{2}$, \\ Alistair Bell ${ }^{2}$, and Jean-Charles Dupont ${ }^{3}$ \\ ${ }^{1}$ LATMOS, IPSL, UVSQ Université Paris-Saclay, Guyancourt, France \\ ${ }^{2}$ CNRM, Université de Toulouse, Météo-France, CNRS, Toulouse, France \\ ${ }^{3}$ LMD, IPSL, École Polytechnique, Palaiseau, France
}

Correspondence: Pragya Vishwakarma (pragya.vishwakarma@latmos.ipsl.fr)

\begin{abstract}
Cloud radars are capable of providing continuous high-resolution observations of the cloud. These observations are related to the microphysical properties of clouds. Power law relations in the form of $Z=a \cdot L W C^{b}$ are generally used to estimate liquid water content (LWC) profiles. The constants a and $b$ from the power-law relation vary with the cloud type and cloud characteristics. Due to the variety of such parameterizations, selecting the most appropriate Z-LWC relation for a continuous cloud system is complicated. Additional information such as Liquid water path (LWP) from a co-located microwave radiometer is used to scale the LWC of the cloud profile. An algorithm for estimating the LWC of warm clouds using radar-microwave radiometer synergy in a variational framework is presented. This method also accounts for attenuation due to cloud droplets and retrieves a suitable scaling factor $(\ln a)$ of the profile in addition to the LWC. The optimal estimation techniques incorporate a priori information of desired variables, and the forward model converts these variables into observation parameters. In this algorithm formulation, the measure of uncertainty in observations, forward model and, a priori acts as weights in the retrieved quantities. These uncertainties in the retrieval are analyzed in the sensitivity analysis of the algorithm. The retrieval algorithm is first tested on a synthetic profile for different perturbations in sensitivity parameters. The sensitivity study has shown that this method is susceptible to LWP information. The algorithm is then implemented to various cloud and fog cases at SIRTA observatory to estimate LWC and the scaling factor. The scaling factor changes for each cloud profile, and the range of lna are consistent with suggested values in literature. The validation of such an algorithm is challenging, as we need reference measurements of LWC co-located with the retrieved values. During the SOFOG-3D campaign (South-West of France, October 2019 to March 2020), in-situ measurements of LWC were collected in the vicinity of a cloud radar and a microwave radiometer, allowing comparison of retrieved and measured LWC. The comparison demonstrated that the cloud-fog heterogeneity was playing a key role in the assessment.

The proposed synergistic retrieval algorithm is applied to 39 cloud and fog cases at SIRTA, and the behavior of the scaling factor is studied. This statistical analysis of scaling is carried out to develop a radar-only retrieval method. The climatology revealed that the scaling factor can be linked to the maximum reflectivity of the profile. From climatology, the statistical relations for scaling factor are proposed for fog and cloud. Thanks to the variational framework, a stand-alone radar version of the algorithm is adapted from the synergistic retrieval algorithm, which incorporates the climatology of scaling factor as $a$
\end{abstract}


priori information to estimate the LWC of warm cloud. This method allows the LWC estimation using only radar reflectivity and climatology of scaling factor.

\section{Introduction}

Low-level clouds cover a significant area globally and contribute to $60 \%$ of net radiative forcing in earth's radiation budget (Hartmann et al., 1992). Among all the uncertainties in climate sensitivity estimates, representation of boundary layer clouds has a significant contribution, specifically in the sensitivity of boundary layer clouds to changing surface and boundary layer properties (Bony and Dufresne, 2005). The impact of clouds on climate is further complicated by feedback mechanisms involving cloud and temperature (Stephens, 2005) and cloud-aerosol interactions (Rosenfeld et al., 2014; Fan et al., 2016). Understanding boundary layer cloud dynamics under changing atmospheric circumstances will help to minimize model uncertainty and climate sensitivity (Bony and Dufresne, 2005). On the other hand, fog and haze are disastrous low-visibility phenomena that occur near the surface which are associated to economic implications.

Active and passive remote sensing instruments are suitable for long-term cloud observations from space and the ground (Zhu et al., 2017). Such space borne (e.g. CloudSat (Stephens et al., 2002), CALIPSO (Winker et al., 2010)) and ground based sensors provide observations of various macro and microphysical properties of clouds at different temporal and spatial resolution (Illingworth et al., 2007). Earlier studies demonstrated the quantification of cloud microphysical parameters such as effective radius $\left(r_{e}\right)$ and cloud liquid water content (LWC) using different parameterization with single or multi sensor observations as input. The mass of water content in each cubic meter of dry air at a given altitude is defined as LWC, which is an important parameter for understanding the cloud lifetime and evolution processes.

At $95 \mathrm{GHz}(3.2 \mathrm{~mm})$, the Rayleigh regime is still valid as the radar wavelength is nearly two orders of magnitude longer than the observed cloud droplet size, which is invariably less than $50 \mu \mathrm{m}$ (Miles et al., 2000). Therefore, radar reflectivity can be considered proportional to the sixth moment of the droplet spectrum and whereas, LWC is proportional to the third moment of the droplet spectrum. However, Mie scattering becomes significant at larger sizes, such as drizzle droplets. An empirical approach of estimating LWC using radar reflectivity factor by assuming the shape of DSDs, is demonstrated in literature. Z-LWC relationships derived using in-situ measured droplet spectra collected from a research aircraft are proposed in Atlas (1954); Sauvageot and Omar (1987); Fox and Illingworth (1997). Table 1 shows details of empirical relations between the

50 radar reflectivity factor $\mathrm{Z}$ and the $\mathrm{LWC}$ from literature for a given cloud type. Typically, radar reflectivity $\mathrm{Z}$ and cloud liquid water content (LWC) are related with a power law equation given as:

$Z=a \cdot L W C^{b}$

where $\mathrm{a}$ and $\mathrm{b}$ are constant coefficients. If $\mathrm{Z}$ is known, LWC can be estimated provided the value of constants $a$ and $b$ are correct for the given cloud type.

LWC calculated using any Z-LWC relationships listed in Table 1 depends strongly on cloud microphysics which varies significantly with changing ambient conditions. Due to the inherent heterogeneity of cloud droplet spectra, it is challenging to 
Table 1. Z-LWC relation from literature

\begin{tabular}{ccccc}
\hline Reference & Z-LWC relation & lna & Cloud type & Assumption \\
\hline Atlas (1954) & $Z=0.048 \cdot L W C^{2.0}$ & -3.0365 & Clouds without Drizzle & Empirical \\
Sauvageot and Omar (1987) & $Z=0.03 \cdot L W C^{1.31}$ & -3.5065 & Non-precipitating stratocumulus and cumulus & Empirical \\
Fox and Illingworth (1997) & $Z=0.012 \cdot L W C^{1.16}$ & -4.4228 & Non-precipitating marine stratocumulus $\quad$ Empirical \\
Baedi et al. (2000) & $Z=0.015 \cdot L W C^{1.17}$ & -4.1997 & Stratocumulus clouds & Empirical \\
Wang and Geerts (2003) & $Z=0.044 \cdot L W C^{1.34}$ & -3.1235 & Non-precipitating marine stratus & Drizzle clouds \\
Krasnov and Russchenberg (2005) & $Z=323.59 \cdot L W C^{1.58}$ & 5.7794 & Empirical \\
\hline
\end{tabular}

establish a universal Z-LWC relationship as the value of coefficients $a$ varies from 0.012 for marine stratocumulus cloud (Fox and Illingworth 1997) to 323.59 for drizzling cloud (Krasnov and Russchenberg 2005), and the exponent $b$ varies from 1 to 2. As mentioned, the empirical approach is also based on certain approximations in DSDs, which widely vary within the cloud and among different cloud systems. Thus, small variation in droplet spectrum strongly influences the Z and LWC relationship and leads to high uncertainties in estimated LWC profile (Löhnert et al., 2001). Since the shape of droplet spectrum changes significantly within the cloud structure, the retrieval of LWC using only Z information will not be accurate even if the most appropriate empirical relation for the cloud type is used.

To reduce the uncertainties due to unknown droplet spectra, a synergy of two or more active and passive sensors providing additional cloud information with sophisticated retrieval techniques has been used in several studies in the past few decades. Some studies demonstrated the applicability of dual-wavelength radar system, which uses signals from the $K_{a}$-W band (Hogan et al., 2005), $S-K_{a}$ band (Ellis and Vivekanandan, 2011) to calculate liquid water profile. Frisch et al. (1995, 1998) used total integrated liquid water path (LWP) measured by microwave radiometer with cloud radar together. LWP is defined as follows:

$$
L W P=\Sigma(L W C) d r
$$

where $d r$ is the range resolution in meters if LWP is in $\mathrm{gm}^{-2}$ and LWC is in $\mathrm{gm}^{-3}$.

This radar-radiometer combination constrained the retrieved LWC exactly to the observed LWP. Further, Ovtchinnikov and Kogan (2000) used cloud simulated data to conclude that combination of radar reflectivity with liquid water path from microwave radiometer can significantly increase the accuracy and the robustness of the retrieval. Thereafter, Löhnert et al. (2001) explained a similar approach of using LWP derived using brightness temperature $\left(T_{b}\right)$ from a passive microwave radiometer, radar reflectivity profile from a $95 \mathrm{GHz}$ cloud radar, and cloud model statistics to derive LWC profiles. The limitation of this approach is that the accuracy of LWC profile is reduced in presence of drizzle. O'Connor et al. (2005) calculated the drop size, liquid water content, and liquid water flux of drizzle using the synergy of cloud radar and backscattering information from lidar. This technique was applied to the drizzle below the cloud base, as lidar beam is strongly attenuated when it penetrates the cloud. 
A retrieval method which is applicable to different liquid water clouds and fog is yet to be proposed. The main goal of this study is to make the most of the LWC retrieval when additional information is available with radar measurement, and utilize this knowledge to improve the LWC retrievals when this additional information is not available. The instrumentation used in this paper is described in section 2 and the retrieval methodology to develop climatology is explained in section 3. Section 4 elaborates the sensitivity analysis of the retrieval algorithm using synthetic profile, and the validation of retrieval with in-situ measurements is discussed in section 5. After evaluating the performance of the retrieval algorithm, section 6 focus on derivation of climatology of the retrieved parameters and finally the BASTA standalone retrieval using climatology is discussed in section 7 .

\section{Observation sites and instrumentation}

Observations for this study are collected from a $95 \mathrm{GHz}$ cloud radar and a microwave radiometer which are co-located in two different locations. The longest observation period, between November 2018 to May 2019 which corresponds to the meteorological conditions of interest including relatively higher concentration of fog and cloudy days, is from SIRTA (Haeffelin et al., 2005, Site Instrumental de Recherche par Télédétection Atmosphérique). SIRTA is a multi-instrumental atmospheric research laboratory located in Palaiseau (49N, 2E), $20 \mathrm{~km}$ south of Paris (France) in a semi-urban environment which is $160 \mathrm{~m}$ above sea level. The observatory brings together several advanced active and passive remote sensing instruments to study the dynamic and radiative processes of the atmosphere recorded since 2002 (Haeffelin et al., 2005). The climatology of liquid cloud retrievals is derived using the observations from SIRTA. Simulations using the French Convective Scale AROME model (Seity et al., 2011; Brousseau et al., 2016) for SIRTA are used for sensitivity analysis of the algorithm.

The second site is located in the South-West of France, measurements were collected during the SOFOG-3D (SOuth west FOGs 3D experiment for processes study) field experiment between October 2019 to March 2020. This field experiment was conducted to advance the understanding of fog processes by exploring both horizontal and vertical variability of fog layers. The super-site is located at Saint-Symphorien commune of France and is centered at $44^{\circ} 24^{\prime} 44.5 \mathrm{~N}, 0^{\circ} 35^{\prime} 51.5 \mathrm{~W}$ covering a circular surface of $5 \mathrm{~km}$ radius around this point. The territory is part of a farm named Domaine de la Grande Téchoueyre which is $69 \mathrm{~m}$ above sea level and this site was chosen due to its fog occurrence statistics. Additionally, various measurements of fog properties were collected with innovative sensors including in-situ and remote sensing networks across a $300 \times 200$ $\mathrm{km}$ domain around the super-site. In-situ measurements collected during this campaign are used to validate the LWC retrieval algorithm in fog conditions. The next part goes into the details about the specifications of instrumentation used in this study.

\subsection{BASTA cloud radar at SIRTA and SOFOG-3D}

A vertically pointing cloud radar called BASTA (Delanoë et al., 2016) is operating at SIRTA to record the time height structure of cloud, fog and light precipitation. BASTA was developed at LATMOS (Laboratoire Atmosphères, Observations Spatiales) and it has been operational at SIRTA observatory since 2011. This Doppler cloud radar uses the frequency-modulated continuous wave (FMCW) technique, rather than pulses, making it less expensive than traditional cloud radars. It measures radar 
reflectivity and Doppler velocity of the atmospheric targets at four different resolution modes depending on the specific application. In particular, the $12.5 \mathrm{~m}$ vertical resolution mode is dedicated to fog and low clouds and is limited to $12 \mathrm{~km}$ range height. The $25 \mathrm{~m}$ mode is suitable for liquid and ice mid-tropospheric clouds and covers the vertical extent from minimum range of $40 \mathrm{~m}$ to $18 \mathrm{~km}$. Furthermore, the $100 \mathrm{~m}$ resolution is ideal for optically-thin high-level ice clouds with maximum detectable range of $24 \mathrm{~km}$. This radar is calibrated using the approach proposed by Toledo et al. (2020) based on corner reflectors. Another product developed by combining three modes providing optimized radar reflectivity, velocity and mask indicating the valid signal from noise is also developed. This level 2 (L2 here onwards) processing is a new vertical grid derived by combining several modes (vertical and temporal resolution) at the same time resolution in order to make the most of each mode. The largest range resolution data is used for their higher sensitivity, and background noise is removed. In this study, 39 cloud cases with L2 product of BASTA measurements at SIRTA location are used. During the SOFOG-3D field experiment, the vertically pointing BASTA radar was deployed at a fog prone region in order to acquire high-resolution observations of the fog's characteristics. The L2 product of BASTA observation is used to evaluate the performance of the algorithm for retrieving LWC of low-level fog. Due to the coupling of the radar antenna the minimum detectable range was $40 \mathrm{~m}$ above the ground.

\subsection{HATPRO microwave radiometer at SIRTA and SOFOG-3D}

A 14-channel HATPRO (Humidity And Temperature Profiler) MWR manufactured by Radiometer Physics GmbH (RPG) is operational at SIRTA observatory. HATPRO MWR is a passive instrument, converting the naturally emitted downwelling radiative energy emitted from the atmosphere within two spectral bands: the first one focuses on the $22.24 \mathrm{GHz}$ water vapor absorption band up to $31 \mathrm{GHz}$ while the second one is centered on the $60 \mathrm{GHz}$ oxygen complex band (51-59GHz). Through the use of calibration coefficients, detected intensities are then directly converted into brightness temperatures. A retrieval technique is then needed to convert the brightness temperature spectra into vertical profiles of temperature, humidity as well as liquid water path. In general, statistical methods (linear, quadratic regressions or neural networks) trained from simulated MWR observations from a database of radiosoundings or model analyses are used (Cimini et al., 2006). Optimal estimation retrievals combining an a priori estimate of the atmospheric state with observations through an iterative process can also be used (Martinet et al., 2020). In this study, LWP retrievals based on MWR observations have been retrieved through quadratic regressions trained from a database of radiosoundings for SIRTA while for SOFOG3D, neural networks trained from AROME short-term-forecasts have been used. MWRs are only sensitive to the total liquid water content present in the vertical profile (Ware et al., 2002). Humidity profiles can be retrieved with a limited vertical resolution due to the smoother weighting functions for K-band channels. Temperature profiles show a better vertical resolution, which can be improved through the use of different elevation angles (generally from 90 to $5.4^{\circ}$ above the ground).

If there is a single layered liquid cloud, MWR thus provide a direct estimate of the LWP for the cloud column. The LWP measurements of the column are unaffected by ice clouds above liquid clouds. The time resolution of LWP measurements used in this study is 1 second, with brief interruptions due to boundary layer scans. The missing measurements during boundary layer scans are interpolated to the BASTA observation frequency. The uncertainty of the MWR for LWP is expected to range 
between $10 \mathrm{gm}^{-2}$ and $20 \mathrm{gm}^{-2}$ (Crewell and Löhnert, 2003; Marke et al., 2016) particularly dependent on the absolute calibration errors of MWR and uncertainties in retrieval algorithms.

\subsection{Cloud Droplet Probe (CDP) on tethered balloon during SOFOG-3D experiment}

The tethered balloon mounted with in-situ sensor called Cloud Droplet Probe (CDP) which is designed to measure cloud droplet size distribution from $2 \mu \mathrm{m}$ to $50 \mu \mathrm{m}$. The CDP probe housing contains the forward scatter optical system, which includes a laser heating circuit, photodetectors, and analog signal conditioning and an appropriate data system can also calculate various other parameters including particle concentrations, effective diameter (ED), Median Volume Diameter (MVD), and Liquid Water Content (LWC) (Lance et al., 2010). This instrument is designed and commercialized by Droplet Measurement Technology and the specifications are given in table 2. The sampling rate of CDP was $10 \mathrm{sec}$ during SOFOG-3D campaign.

Table 2. Specifications of in-situ cloud droplet probe mounted on tethered balloon

\begin{tabular}{cc}
\hline Laser & $658 \mathrm{~nm}$, up to $50 \mathrm{~mW}$ \\
Measured Particle Size Range & $2 \mu \mathrm{m}-50 \mu \mathrm{m}$ \\
Typical Sample Area & $0.24 \mathrm{~mm}^{-2}$ \\
Number Concentration Range & $0-2,000 \mathrm{~cm}^{-2}$ \\
\hline
\end{tabular}

\section{Methodology of LWC retrieval}

The objective of the algorithm is to retrieve $L W C$ using radar reflectivity measurements and $L W P$ derived from MWR when the latter is available. The integrated liquid water content in the cloud column constrains the vertical profile of $L W C$ which is strongly related to reflectivity profile. There are several methodologies for modeling such algorithms, including analytical methods, machine learning techniques, and others. The technique proposed in this paper is framed within the context of optimal estimation theory (Rodgers, 2000). This approach combines a priori information and uncertainties in the observations, the way we represent them and is easily expandable to accommodate additional information from multiple instruments. This retrieval method must be able to combine active and passive remote sensing instruments to derive the most possible accurate climatology of liquid cloud properties and also work when only radar observations are available (i.e. stand alone version). This must be achieved using a common framework. Such a technique has been widely applied in previous studies (Löhnert et al., 2001; Hogan, 2007; Delanoë and Hogan, 2008). Synergistic retrieval combining radar and microwave radiometer in order to estimate liquid cloud properties has been already proposed by Löhnert et al. (2001). In their approach, they directly assimilate brightness temperature $\left(T_{b}\right)$ and humidity profiles from microwave radiometer. The method presented here aims at providing more flexibility when the microwave is not available. Therefore, we do not directly assimilate brightness temperatures but the microwave radiometer product $(L W P)$ and the associated uncertainties are taken into account. In standalone mode, when only 
https://doi.org/10.5194/amt-2022-3

Preprint. Discussion started: 31 January 2022

(c) Author(s) 2022. CC BY 4.0 License.
Atmospheric

Measurement

Techniques

Discussions

radar is available, our method relies on a priori knowledge of liquid cloud properties and their link with radar measurements.

This a priori information will be built using climatology derived when radar and microwave radiometer are simultaneously available.

Figure 1, which represents the block diagram of the method illustrates how the input parameters ( $\mathrm{Z}$ and LWP) are used to retrieve the output variables ( $L W C$ and $\ln a$, where lna comes from the power law relation Z-LWC in Eq.1), will support the discussion in the next sections.

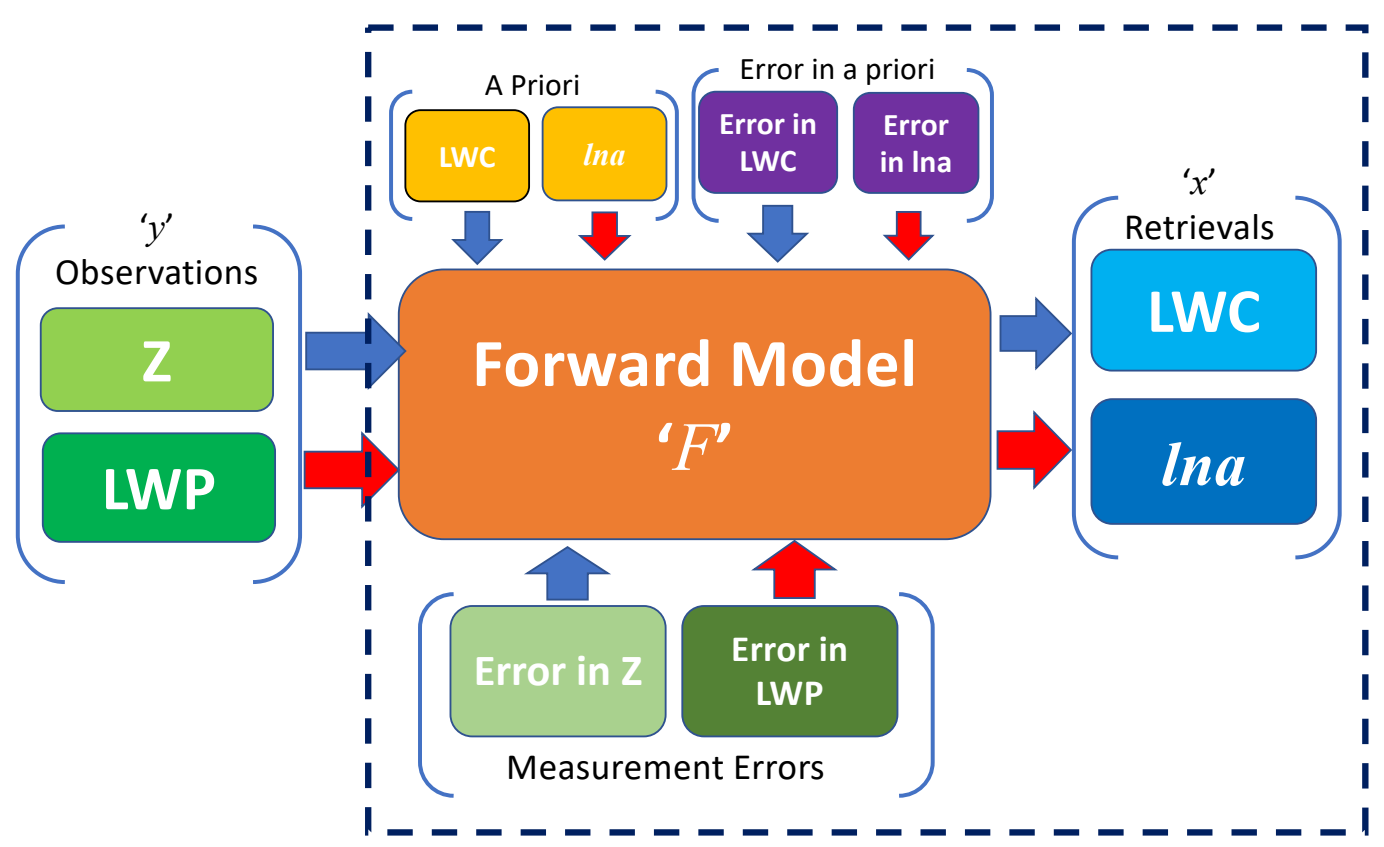

Figure 1. Schematic of LWC retrieval algorithm.

\subsection{Optimal estimation formulation}

The optimal estimation (Rodgers, 2000) is a retrieval approach in which the measured quantities are related to unknown atmospheric parameters using a Forward model. If ' $y$ ' is the measurement and ' $x$ ' is the unknown parameter, then the forward model ' $F$ ' and errors ' $\epsilon$ ' can be mathematically written as

$y=F(x)+\epsilon$ 
where error due to measurements and forward model are accounted in $\epsilon$. The forward model is a mathematical description of the atmosphere as a function of the measurements and the atmospheric states. From Eq. (3), to retrieve $x$ (atmospheric states) as $x=F^{-1}(y-\epsilon)$, it is essential to have good knowledge of $F$ before physically inverting it because such operators are generally complex and non-invertible. This problem is referred as the inverse problem. An example of solution of inverse problem is a probabilistic optimization based on Gauss-Newton method (Rodgers, 2000) by minimizing the cost function $J$ as:

And the forward model is linearized about the state vector $x$ at $i^{t h}$ iteration, then $y$ can be written as

$y=F\left(x_{i}\right)+K_{i}\left(x-x_{i}\right)$

Minimization of cost function leads to iterative solution for the state at $i+1$ iteration

$x_{i+1}=x_{i}+A^{-1}\left[K^{T} R^{-1} \delta y+B^{-1} \delta x_{a}\right]$

where $A^{-1}=K^{T} R^{-1} K+B^{-1}$ gives the error covariance matrix of optimized solution of $x$ after convergence is achieved. $\mathrm{K}$ is called the Jacobian matrix, containing the partial derivative of measurement with respect to state parameter. $R$ is the error covariance matrix accounting for observation and forward model errors, $B$ is the error covariance matrix for 'a priori' information and $x_{a}$ denotes the a priori of the state vector. A prior or background information of the unknowns (generally derived from the climatology or model), is used to constrain the inverse problem. The retrieval starts with the 'first guess'(can be a priori) of the states and the forward model is then applied to simulate the values of measurements. The states are updated until the simulated and measured quantities are close enough and convergence is achieved.

Convergence is assessed at each iteration using the following variable to estimate the closeness of the observations with the model:

$G=|J(i)-J(i-1)|$

where ' $i$ ' is the iteration number.

For every iteration, $G$ examines the absolute gradient of cost function and achieves the convergence when the difference between two successive cost functions is negligible. In this scenario, the retrieval converges when $G$ is of the order of, $10^{-7}$ which indicates that the additional iteration is not adding prominent change in the retrievals.

\subsection{Definition of the state and observation vectors}

The state vector ' $X$ ' is the vector of unknowns, must contain all the variables to retrieve. The observation vector ' $Y$ ' is driven by the available observations. In our case, the radar reflectivity and $L W P$ (when microwave radiometer is available) are the parameters in the observation vector. These two vectors are also defined in the way that we can link them through the forward model. The forward model accounting for radar attenuation will be described in details in section 3.3. 
From the power law relation of Z-LWC in Eq. (1) the constants $a$ and $b$ are dependent on many microphysical parameters such as the particle size, number concentration and other ambient conditions. Through this kind of relationships we can associate a $L W C$ value to a reflectivity value, adding $L W P$ retrieved by the microwave radiometer we can release one more constrain and adjust one of the parameters of the Z-LWC relationship that varies with each profile. This is because the retrieved LWC is constrained by the observed LWP. The choice of using the pre-factor $a$ is motivated by its capability to adjust the whole profile of $L W C$ regardless the reflectivity and $a$ shows a much higher variability than $b$. Note that the impact of variability in $b$ will be assessed in section 4.1 .

The state and observational vectors are defined as follows:

$$
\begin{gathered}
X=\left(\begin{array}{c}
\ln L W C_{1} \\
\vdots \\
\ln L W C_{n} \\
\ln a
\end{array}\right), \\
Y=\left(\begin{array}{c}
\ln Z_{1} \\
\vdots \\
\ln Z_{n} \\
\ln L W P
\end{array}\right)
\end{gathered}
$$

To account for the large dynamic range of the observations within a profile, this algorithm uses the logarithm of the state variables and measured quantities which also prevent the unrealistic retrieval of negative values.Therefore, the linear relation between $\mathrm{Z}$ and LWC in $\log$ space in the form of, $y=m x+c$ where lna represents intercept and $b$ is the gradient of the line can be written as:

$\ln Z=\ln a+b \times \ln L W C$

The logarithm of a priori coefficient $a$ is referred to as scaling factor, and logarithm also enables visualizing the wide range of $a$. Although, the observation vector $y$ may not incorporate LWP when it is unavailable, however by adding the LWP in the observation with $\mathrm{Z}$, the forward model allows retrieving lna in addition to LWC.

The state and observation vectors are defined as shown in Eq. (7) and (8). The errors in measurement are tested using a synthetic profile of observations, and detailed in the section 4.1.The most suitable error in observation vector is set as $25 \%$ and $10 \%$ respectively for $\mathrm{Z}$ and LWP. As mentioned in section 2.2, LWP estimates from MWRs have an expected uncertainty of $\pm 20 \mathrm{gm}^{-2}$. However, this uncertainty estimation also depends on the MWR calibration and retrieval algorithm uncertainties, an approximate evaluation of the LWP measurements using longwave radiation measurements demonstrates an RMSE in LWP of around 5-10 $\mathrm{gm}^{-2}$ during fog with LWP $<40 \mathrm{gm}^{-2}$ (Wærsted et al., 2017). Thus, to minimize the errors due to the measurement uncertainties, the LWP is assimilated only when the measured LWP is greater than $10 \mathrm{gm}^{-2}$ because the relative 
error for low LWP values from HATPRO is significantly higher than for high LWP values. Although, $10 \%$ error in LWP is very small when compared to expected error, but the profiles with LWP values below $10 \mathrm{gm}^{-2}$ are already excluded from retrievals, implying that there is less error to be considered. A detailed analysis of errors in measurement of $\mathrm{Z}$ and LWP are explained in section 4.1, covering the sensitivity analysis of retrieval algorithm using synthetic profile.

Prior knowledge of the state parameters enables the retrieval to be constrained in order to avoid unrealistic solutions, especially when additional measurements are missing. a priori information usually consists of long-term climatology or model outputs of state parameters, i.e. LWC and lna. For example, from various in-situ measurements of LWC in fog or liquid cloud it is known that LWC in the cloud is not equally distributed vertically and is strongly related to reflectivity. A priori of LWC dependent on reflectivity should be more suitable than a constant LWC profile. In this retrieval, a LWC profile derived from the empirical relation is used as the a priori with an a priori error of $1000 \%$ (or 10) for both LWC and lna. Note that the errors are presented in logarithm and the error in the a priori is considered high, because LWP measurements are available to constrain the retrievals. Even so, a priori information is vital in case of missing LWP measurements, which plays an important role in case of LWC retrieval using only radar observations and climatology. In such a case, expected error in the a priori will be considered less. In case of low LWP observations, retrieval depends on a priori which is taken from Atlas (1954) empirical relation and therefore, the scaling factor is not retrieved for such profiles. The retrieval of LWC for the profiles with LWP $<10 \mathrm{gm}^{-2}$ incorporates attenuation in the retrievals, rather than just applying empirical relationships.

\subsection{Description of the forward model and Jacobian matrix}

The forward model is an approximation of the physical phenomenon represented as a function of measurement and state variables. In order to expand the retrieval when additional measurement is available, it is recommended to describe the forward model for each element of the observation vector. The forward model for radar links radar reflectivity to LWC using the Eq. (9). Furthermore, LWP as additional information constrains LWC using Eq. (2) and allows the retrieval of scaling factor lna. When additional information is unavailable, the retrieval constrains LWC using lna climatology, which is elaborated in section 7. The microphysical model for attenuation consideration is discussed in next subsection 3.3.1.

\subsubsection{Forward model for attenuation correction}

Water vapor and oxygen are the two primary atmospheric gases that contribute to microwave absorption. Even though W-band radars work in one of the water vapor transmission windows, absorption due to water vapor can exceed $1 \mathrm{dBkm}^{-1}$ depending on temperature and humidity in the lower troposphere. Despite the fact that attenuation by atmospheric gases is relatively small, attenuation due to liquid clouds droplets can diminish the advantages of W-band radar observation, particularly in the liquid cloud case. According to Lhermitte (1990), the attenuation due to liquid droplets is more problematic as it depends on drop size distribution, which is not known in general. Since attenuation due to liquid cloud is dependent on temperature and density of cloud droplets and clouds consists of randomly distributed, spherical droplets of diameter less than 100 microns, the $95-\mathrm{GHz}$ microwave absorption can be adequately described by the Rayleigh approximation. Various theoretical studies have been conducted to determine the attenuation due to liquid cloud and drizzle at different temperatures. For example, at 
$10^{\circ} \mathrm{C}$, Lhermitte (1990) calculated $4.2 \mathrm{dBkm}^{-1}$ per $\mathrm{gm}^{-3}$ of liquid water attenuation, while Liebe et al. (1989) obtained $4.4 \mathrm{dBkm}^{-1}$ by using the Rayleigh approximation. On the other hand, Vali and Haimov (2001) assumed spherical hydrometeor and obtained the general solution for absorption (and scattering) at W-band using Mie approximation. Extinction due to liquid cloud at $95 \mathrm{GHz}$ using simultaneous and co-located cloud measurements of drop-size distribution, liquid water content, temperature, and pressure for maritime stratus clouds was comparable with the theoretical studies mentioned above. This study further concludes that, for around $10^{\circ} \mathrm{C}$ and pressures close to $900 \mathrm{mb}$, the one-way attenuation ' $\mathrm{A}$ ' in $d B \mathrm{~km}^{-1}$ was found to be linearly dependent on LWC, and expressed as:

$A=0.62+4.6 \times L W C$ in $d B k m^{-1}$,

where $0.62 \mathrm{dBkm}^{-1}$ represents gaseous absorption.

Vivekanandan et al. (2020) calculated attenuation 'A' as a function of reflectivity Z for cloud droplets and drizzle using power law fit. Reflectivity and attenuation are simulated using DSDs collected from VOCALS field experiment (Wood et al., 2011), with $\mathrm{Z}$ being proportional to sixth moments and attenuation being proportional to third moments of DSDs. The DSDs for cloud and drizzle droplets are separated by $-17 d B Z$ threshold for simulated reflectivity and therefore, as given by Eq. (11) and (12) for clouds and drizzle respectively.

$A=18.6 \times Z^{0.58} \mathrm{~dB} / \mathrm{km}$

$A=1.68 \times Z^{0.9} \mathrm{~dB} / \mathrm{km}$

However, even with power law fit, the range of attenuation calculated is 0 to $4 \mathrm{dBkm}^{-1}$, which is almost the same order of attenuation per kilometer calculated using linear relations proposed in previous studies. Equation (10) is used to calculate attenuation due to liquid water in the forward model. As this study is focusing over of retrieval of LWC and its climatology, attenuation as a function of LWC, will adjust with retrieved LWC for cloud and drizzle without categorizing the hydrometeor on the basis of forward modelled reflectivity. It is worth noting that all the attenuation relation mentioned above were derived using DSDs collected from marine clouds, and the calculation of attenuation relation for continental clouds is prospective. Finally, a sensitivity test for considering inconsistent attenuation in the forward model will be discussed in section 4.3.

The attenuation correction is achieved within the forward model by correcting at a particular gate to estimate the associated attenuation, and then using it to correct at all subsequent gates. Therefore, the forward model estimates the two-way attenuation corresponding to LWC using Eq. (10), and then corrects the forward modelled reflectivity to account for the estimated attenuation. Since the radar is vertically pointing, it is presumed that the lowest gate (closest to the radar) remains unattenuated due to the liquid droplets, whereas all gates above are affected by liquid droplets present in the preceding gates. As the radar beam passes through the cloud profile it gets attenuated due to liquid, as a result the top most cloud pixels of the profile are the most attenuated. To summarize, each cloud pixel is corrected for the two-way attenuation caused by liquid clouds along the path of the radar beam. 


\subsubsection{The Jacobian formulation}

The Jacobian is a matrix representing the sensitivity of the forward model. It consists of partial derivatives of all the element of $Y$ vector with respect to $X$ vector. Since the forward model update the element of measurement vector at each iteration, thus, at each iteration step the Jacobian $K$ is re-evaluated and for a profile of ' $n$ ' cloud pixels as

$$
K_{i}=\left(\begin{array}{cccc}
\frac{\partial \ln Z_{1}}{\partial \ln L W C_{1}} & \cdots & \frac{\partial \ln Z_{1}}{\partial \ln L W C_{n}} & \frac{\partial \ln Z_{1}}{\partial \ln a} \\
\vdots & \ddots & \vdots & \vdots \\
\frac{\partial \ln Z_{n}}{\partial \ln L W C_{1}} & \cdots & \frac{\partial \ln Z_{n}}{\partial \ln L W C_{n}} & \frac{\partial \ln Z_{n}}{\partial \ln a} \\
\frac{\partial \ln L W P}{\partial \ln L W C_{1}} & \cdots & \frac{\partial \ln L W P}{\partial \ln L W C_{n}} & \frac{\partial \ln L W P}{\partial \ln a}
\end{array}\right)
$$

$K$ consist of $(n+1) \times(n+1)$ elements with top $n \times n$ elements are partial derivative of reflectivity with LWC and last row corresponds to constrain LWC at each cloud pixel with total LWP. The $(n+1)^{\text {th }}$ column corresponds to the relation between radar reflectivity and scaling factor $(\ln a)$ and the very last element is set to zero because $\ln a$ is not related to LWP measurements. Therefore, for $n$ cloud pixels in a profile, the forward model will evaluate a Jacobian of $(n+1) \times(n+1)$ to retrieve the state vector corresponding to radar reflectivity and LWP measurements. The attenuation in forward modelled reflectivity due to liquid cloud droplets is accounted at every iteration. The Jacobian matrix incorporates the two-way attenuation ' $A$ ' at each cloud pixel by calculating the partial derivatives of 'A' with respect to LWC at each cloud pixel. It is worth noting that the attenuation due to gaseous absorption is not accounted in the Jacobian matrix because L2 reflectivity is already corrected for it. The value of attenuation corresponding to the lna parameter is assumed zero.

The forward model errors are the errors associated to the mathematical model which relate measurements with atmospheric physical parameters. The relationships described in the forward model are not necessarily perfect and hence incorporate error in the retrieval. As mentioned already, $\mathrm{Z}$ is closely related to LWC of cloud and hence forward model for reflectivity is represented by Eq. (9). In this equation, the errors in $\mathrm{Z}$ are taken into error in measurement for $\mathrm{Z}$, whereas $\ln a$ and $L W C$ are retrieved parameters. As exponent $b$ is taken constant, there is a possibility to incorporate error in forward model due to $b$, which is discussed in sensitivity analysis in section 4.5. The error incorporated because of model representation of attenuation due to liquid cloud is also discussed in sensitivity analysis. The cloud liquid water is also constrained by LWP as the summation of LWC for the given cloud column, as shown in Eq. (2). Therefore, the forward model for LWP is simple and therefore, error in the estimation of LWC due to forward model is neglected.

\subsection{Discussion of the retrieval uncertainty}

Other sources of error in the retrieval algorithm are discussed in this section. Doppler radars also detect boundary layer insects, large dust particles and pollens suspended in the air as a result of the convective boundary layer that grows in the morning hours and matures shortly after the midday (Geerts and Miao, 2005). These so-called air-borne planktons developed due to onset of convective boundary layer, contaminate the reflectivity profile. Therefore, the unwanted signal in the radar reflectivity due to airborne planktons must be removed before estimating LWC. Additionally, all the cloud above $2500 \mathrm{~m}$ are anticipated to be mixed phase or ice cloud which cannot be addressed in the same way as liquid cloud and therefore clouds above $2500 \mathrm{~m}$ 
are excluded. The data set employed in this study indicates that the majority of the liquid cloud are observed below $2500 \mathrm{~m}$.

However, because the height of the melting layer changes with season and geographical location, it would be appropriate to determine the height of melting layer to differentiate liquid and mixed phase clouds. As the LWP measurements from MWR are unaffected by overlying ice cloud, but accounts for liquid in the overlying mixed phase cloud, which adds error in the LWC retrieval. Therefore, all such cloud profiles are removed before deriving climatology. The profiles with LWP less than or equal to $10 \mathrm{gm}^{-2}$, the retrieved LWC is not used for climatology due to high relative error in low LWP values.

Fog on the other hand, causes droplet deposition on the radome and hence contributes towards substantial amount of attenuation in the radar reflectivity which is not accounted in the retrieval. It is worth noting that a blower to remove the droplet deposition on BASTA at SIRTA is installed since 2019 which has substantially reduced the wet radome attenuation after rain. Although, the retrieval assumes completely dry radome for all the cases, including clouds immediately after rain and drizzle. Since the retrieval algorithm deals with two independent measurements and therefore the two instruments have distinct observation frequency which is addressed by interpolating the LWP measurements into the radar temporal resolution and hence acts as additional source of error.

Due to the coupling of transmitting and receiving antennas of radar, the vertically pointing radar misses a few lowest gates close to the ground. These unavailable gates do not impact the information about the clouds aloft, but the missing information of thin fog causes over estimation in LWC for the first few available gates. The overestimation is due to the fact that retrieval forces the assimilated LWP of the profile by constraining it over available range gates and hence overestimates the LWC for available gates. The most appropriate way to overcome this issue is to use scanning radar, but for vertically pointing radar we assume that the properties of fog remain the same between the first available gates and the ground, and thus reflectivity is extrapolated (extended) downwards for the unavailable range gates. The extension of range gates is particularly significant for SOFOG-3D experiment cases, which are specifically concerned with fog processes. However, the extension of range gates may introduce inaccuracy into LWC retrieval for fog, as the reflectivity of fog at the surface is not always equal to the reflectivity of the first available gates, particularly for dissipating fog.

\subsection{Analysis of the method when microwave radiometer is available}

This section describes the analysis of retrieval when applied to various cloud types. As detailed in section 3, the retrieval technique is applied to reflectivity data from $95 \mathrm{GHz}$ BASTA radar with LWP estimates from co-located RPG HATPRO microwave radiometer for various cloud cases from SIRTA. Between November 2018 to May 2019, 39 cloud and fog cases at SIRTA observatory are selected to address the algorithm's implementation on warm clouds. The data set contains a relatively large number of cloudy cases, including fog and light drizzle. A detailed discussion of retrieval and algorithm implementation is elaborated for a typical example of cloud in the next subsection.

\subsubsection{Illustration of retrieval of 05 February 2019 case at SIRTA}

360 A case study of one of the selected cloudy cases from SIRTA on 05 February 2019 is presented in figure 2. Figure 2(a) and (b) presents the time height plot of radar reflectivity and velocity respectively. LWP estimated by the radiometer alone through 

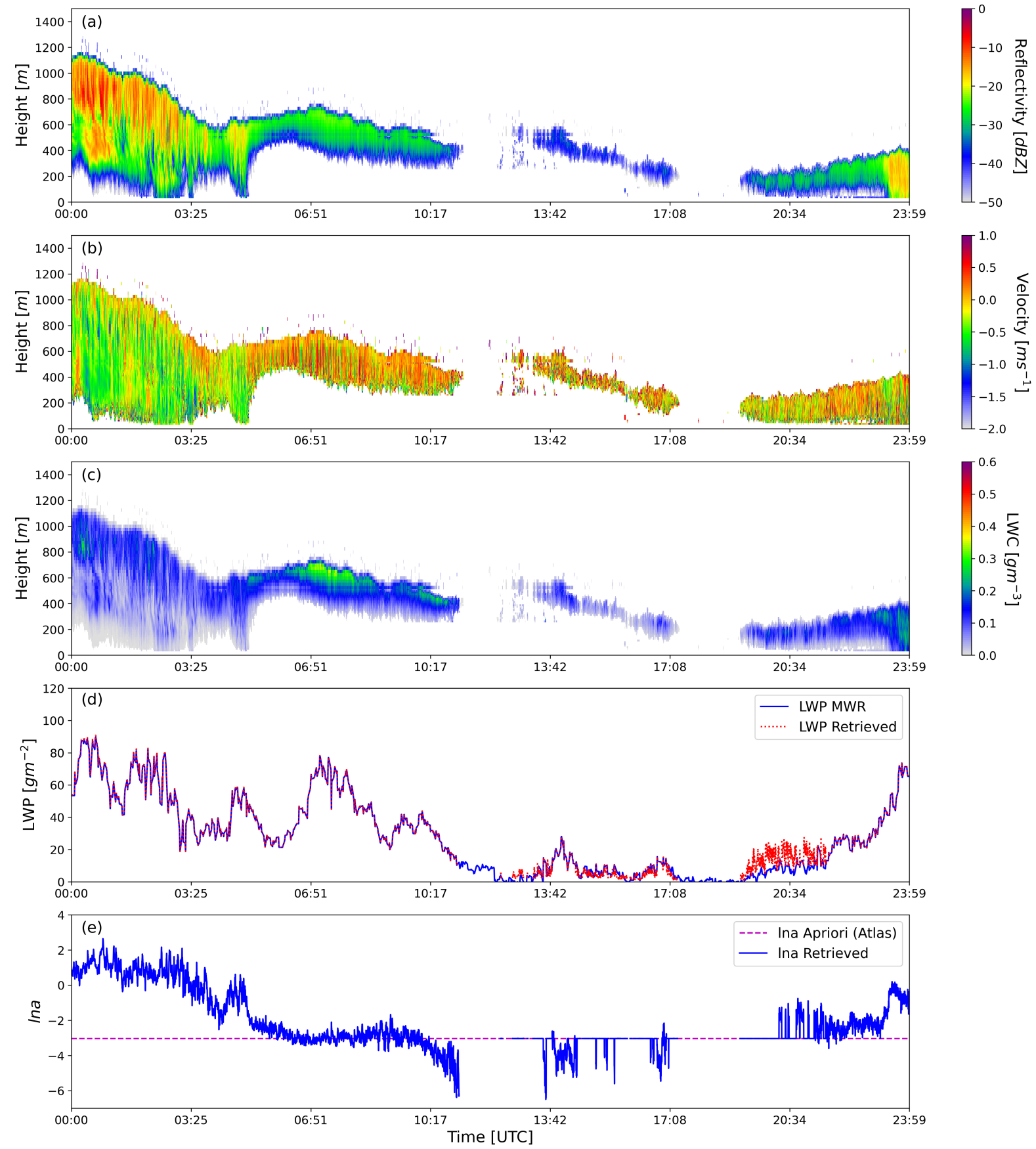

Figure 2. (a) Radar reflectivity (b) Vertical velocity (c) Retrieved LWC, (d) LWP and (e) Retrieved lna for 05 February 2019 case at SIRTA.

quadratic regression is interpolated at radar time of observation as shown in figure 2(d). The retrieved LWC for the cloud pixels is plotted in figure 2(c) and the retrieved scaling factor for each profile is shown in figure 2(e). 
There were no overlapping clouds observed in this instance, and the airborne planktons were removed manually. A dense cloud from midnight with cloud base close to ground dissipates before noon and the formation stage of a fog is initiated after the sunset. The liquid water path remains below $100 \mathrm{~g} \mathrm{~m}^{-2}$ throughout the day. The radar Doppler velocity, displayed in figure 2(b), shows variation in the velocity of the cloud droplets, ranging from -1.5 to $1 \mathrm{~ms}^{-1}$. Within the cloud, the velocities are typically lower and decrease toward the cloud top, when they approach $0 \mathrm{~ms}^{-1}$. Cloud droplets have terminal velocities of only a few centimeters per second, when drizzle droplets develop, the terminal fall velocity increases. Reflectivity values reach $0 d B Z$ for a few profiles indicating drizzle in the beginning (between 00:00 to 03:00 hrs). As indicated by radar observations, higher reflectivity values due to drizzle, yet LWP is nearly identical for the cloud with reflectivity as low as $-35 d B Z$ and contributes the least to LWP. This also explains why it is critical to have LWP information to constrain LWC retrievals, particularly for profiles with drizzle within the cloud and when it evaporates fully before reaching the ground. Figure 2(c) indicates a general increase in LWC towards the cloud top, and the retrieved LWC is less than $0.3 \mathrm{gm}^{-3}$. The scaling parameter has a wide range from -6 to +3 which supports empirical values of $a$ in table 1 . The value of lna changes for each profile. Therefore, this case illustration shows that the retrieval of LWC and scaling factor can be utilized to derive a climatology of scaling factor for different cloud types. It is worth noticing that the retrieval algorithm deals with all the variations of cloud types, and the behavior of scaling factor must be studied. The next section elaborates the robustness of the retrieval algorithm for various sensitivity parameters.

\section{Sensitivity analysis of retrieval algorithm using synthetic data}

The goal of this section to verify the consistency of the retrieval behavior and to assess the sensitivity of the algorithm to inputs, errors and hypotheses. Sensitivity analysis does not replace a proper validation of algorithm retrievals, in section 5 a comparison with in-situ measurement is discussed. Like every other algorithm, this retrieval algorithm also suffers from some fundamental uncertainties which must be addressed. To do so, we use a sensitivity analysis approach. It can also be referred to as 'what-if' analysis, where the input parameters of the model are varied one by one. As shown in the schematic of the retrieval algorithm in figure 1, the retrieval is sensitive to not only input parameters but also other settings like the a priori, expected errors in measurement and a priori information. To quantify the sensitivity of the retrieval algorithm, real observations are not used because the true profile of LWC from an in-situ sensor is not always available. Instead, synthetic data which contains all the characteristics of real observations are used to evaluate the performance of the algorithm. Maahn et al. (2020) highlighted major benefits of using synthetic data to test algorithms and models. First and foremost, systematic forward model errors cancel each other, and second, we know the true atmospheric state $X_{\text {truth }}$, which can be compared with the retrieved optimal result $X_{r e t}$. Hence, considering the mentioned advantages, we are using synthetic data for the sensitivity analysis of the retrieval algorithm.

The flowchart of sensitivity analysis is presented in figure 3 where sensitivity parameters are the parameters in the retrieval algorithm which are perturbed, and the impact is tested. The objective is to formulate input parameters from truth and by feeding synthetic observation to the retrieval algorithm, the result should match with the truth. In the block diagram, synthetic 
https://doi.org/10.5194/amt-2022-3

Preprint. Discussion started: 31 January 2022

(c) Author(s) 2022. CC BY 4.0 License.
Atmospheric

Measurement

Techniques

Discussions

observations ( $\mathrm{Z}$ and LWP) are fabricated using the forward model. The block inside the dashed line is the same as shown inside the dashed line in figure 1 with all the sensitivity parameters.

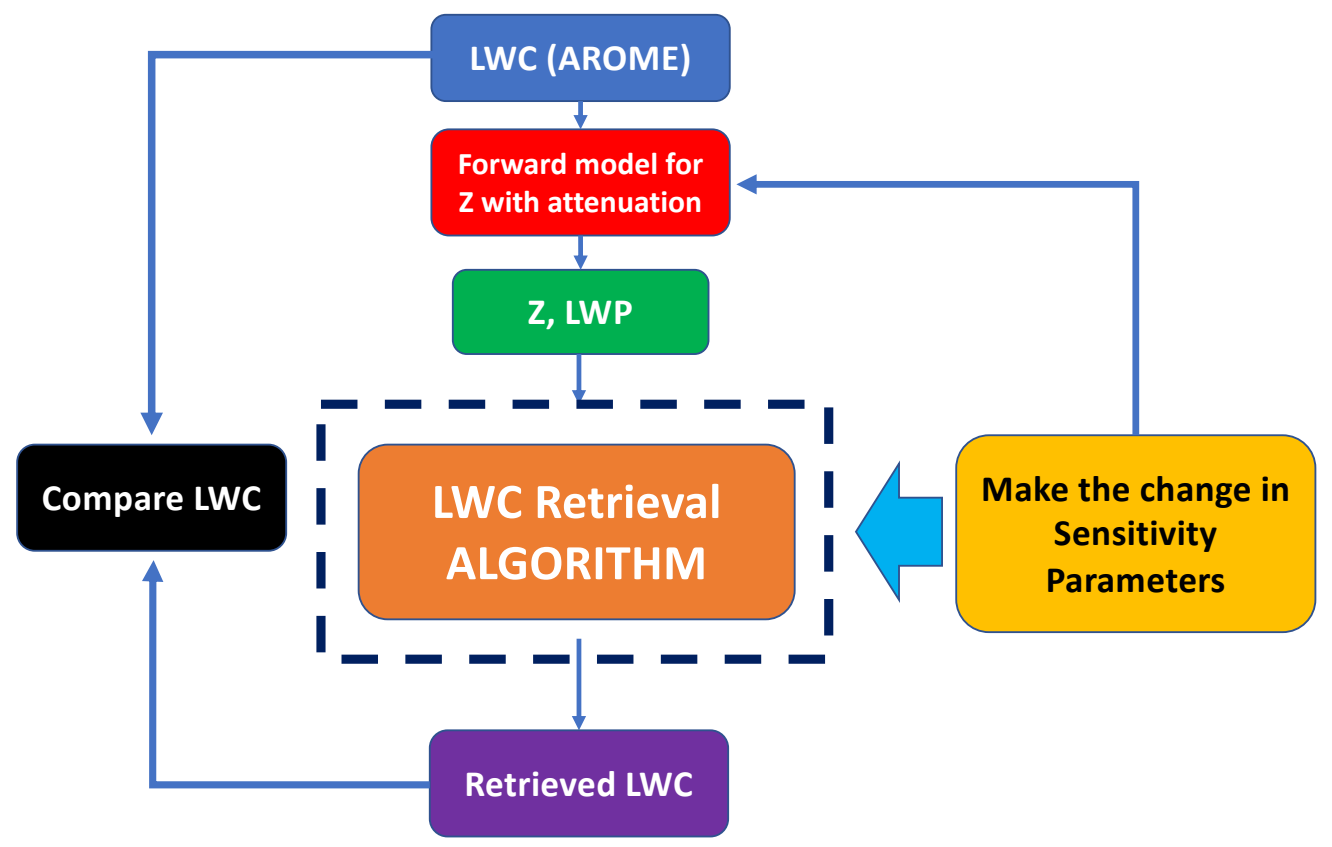

Figure 3. Flow chart for sensitivity analysis of retrieval algorithm.

However, we are aware of the fact that the retrieval errors might be different when observed in real observation scenario, which are already discussed in the section 3.3 for real observations. The error in retrieved LWC from with respect to what we consider as true LWC is calculated using the Eq. (14), (15), and (16) for all the sensitivity test.

1. Root mean squared error

$$
R M S E=\sqrt{\frac{\sum_{0}^{n}\left(L W C_{r e t}-L W C_{\text {true }}\right)^{2}}{n}}
$$

2. $R^{2}$ (coefficient of determination) quantifies the degree of any linear correlation between observations $\left(L W C_{t r u e}\right)$ and retrievals ( $L W C_{r e t}$ ). The general definition of $R^{2}$ regression score function is:

$$
R^{2}=1-\frac{S S_{r e s}}{S S_{t o t}}
$$


where $S S_{\text {res }}$ is residual sum of squares and $S S_{t o t}$ is total sum of squares.

3. Mean absolute percentage error: It measures the accuracy of the retrieval in percentage.

$$
M A P E=\frac{100}{n} \Sigma_{0}^{n}\left|\frac{L W C_{\text {true }}-L W C_{\text {ret }}}{L W C_{\text {true }}}\right|
$$

where $L W C_{\text {ret }}$ and $L W C_{\text {true }}$ are retrieved and true LWC respectively, and $n$ is the number of data points. Analysis of each sensitivity parameter is presented in the next section.

\subsection{Description of synthetic data}

Synthetic data of LWC can be prepared from empirical relations, satellite observations, theoretical adiabatic LWC or model forecasts. For this sensitivity analysis, we opted to include physical parameters of 16 November 2018 fog structure simulated by the AROME model of the retrieval algorithm. The selection requirement for this instance is that it contains a sufficient number of LWC profiles to evaluate the behavior of the algorithm.

AROME is a French convective scale NWP model, operational since 2008 covering France and western Europe providing high-resolution simulations of fog forecasts at $1.3 \mathrm{~km}$ of horizontal resolution and 90 vertical levels of 144 profiles. Detailed setup of the AROME model and fog forecast is explained in Bell et al. (2021). LWC of a fog structure from AROME shortterm forecasts at the nearest grid location of SIRTA is considered as the true atmospheric state. In this case, we are considering only liquid droplets, with no overlapping of liquid or ice clouds aloft. Profiles of true LWC are used to synthesize observation parameters like radar reflectivity using the previously defined power law relation and the liquid water path of each profile by integrating true LWC at each pixel. The forward model (block in red) consisting of the power law relation and attenuation correction model for deriving the synthetic profile of $Z$ using coefficients $a$ and exponent $b$ is taken from Atlas (1954) the empirical relation. The two-way attenuation correction applied to $\mathrm{Z}$ is calculated from Eq. (10). Figure 4(a) shows the distribution of true LWC as a function of time and height, and the synthetic profile of $\mathrm{Z}$ is plotted in figure 4(b) and in figure 4(c) LWP calculated by integrating true LWC.

One of the most obvious sources of uncertainty in the retrieval is the observation (calibration errors and instrumental noise) and forward model errors. The forward model errors tested in this sensitivity analysis are the variation in attenuation consideration and the variation in exponent $b$. As the observation vector, $Y$ contains measurements from two independent instruments, bringing random and uncorrelated errors within the elements of Y (Maahn et al., 2020). The deposition of liquid droplets on the radome introduce an additional bias in radar observations. This is tested by analyzing the impact of possible biases in $\mathrm{Z}$. The next sections cover the sensitivity analysis of the retrieval algorithm for perturbations in different parameters.

\subsection{Sensitivity analysis of impact of error in observation}

435 The input for synergistic retrieval in the observation vector $Y$ consists of concatenated observations from the cloud radar and the radiometer. Each instrument has different errors, and it is worth mentioning that in case of radar observations, instrumental errors are considered for each gate whereas for the LWP measurement from the radiometer the observation error is estimated 

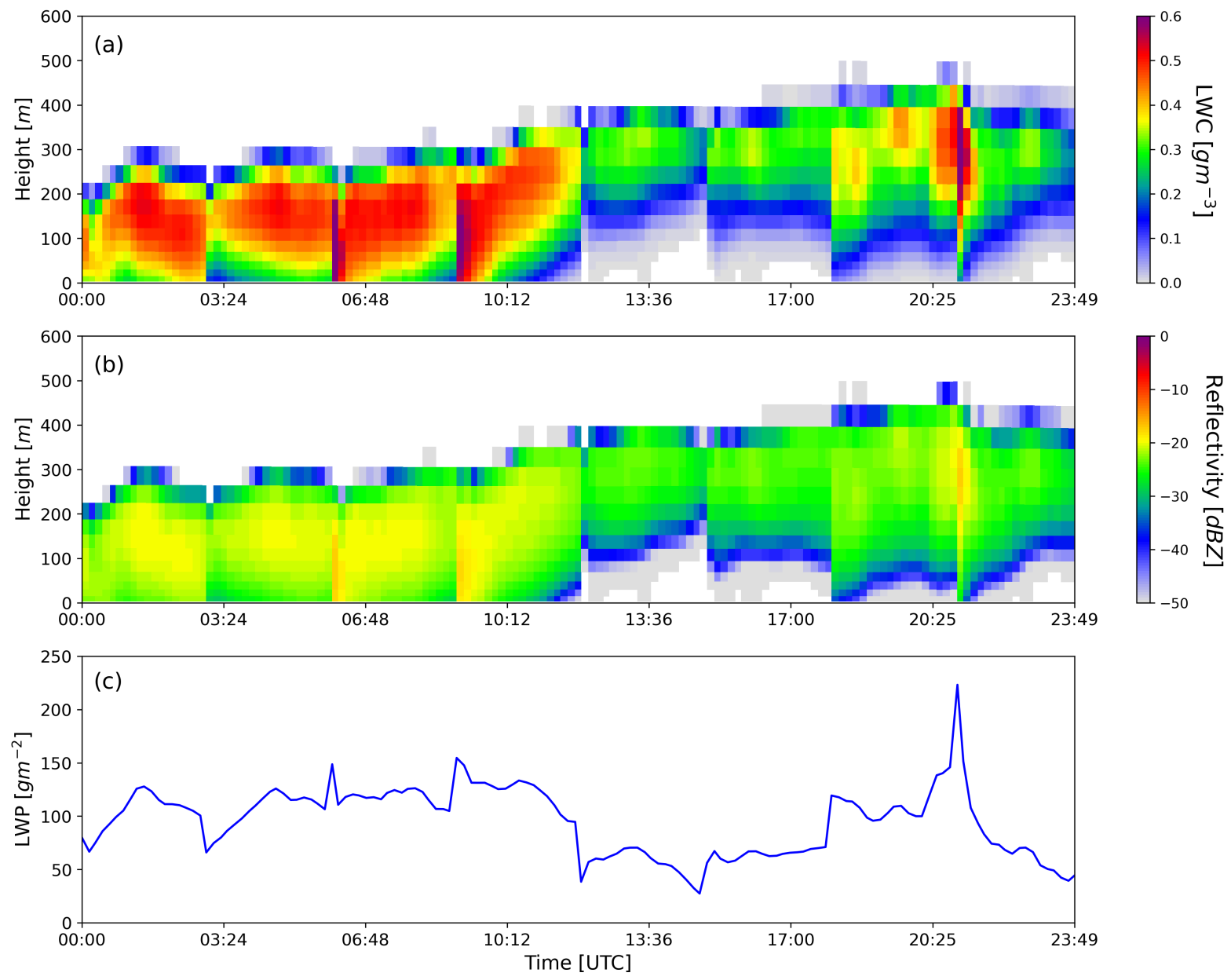

Figure 4. Simulations from AROME model for 16 November 2018 showing (a)True LWC in $\mathrm{gm}^{-3}$, (b) Synthetic reflectivity and (c) LWP.

over the entire cloud profile i.e. an integrated measurement. By varying the weight of instrumental error from each observation ( $\mathrm{Z}$ and LWP) and keeping the rest constant, impact on the retrieved LWC is compared with the true LWC.

Observation errors are assumed to be independent, and the synthetic observations of $\mathrm{Z}$ and LWP are calculated using true LWC, as shown in figure 4. Equation (10) is used to calculate attenuation due to liquid water in the synthetic profile as well as in the forward model. A priori for LWC is calculated using synthetic reflectivity and scaling factor from empirical relation proposed by Fox and Illingworth (1997). Since we are looking at the impact of observation error, the retrieved parameters should have the least contribution from a priori and therefore high error in a priori (1000\% in this case) is considered. Because a priori of LWC is calculated from synthetic $\mathrm{Z}$, a priori of LWC must be different from true LWC to minimize the contribution of a priori which forces retrieval to be close to true LWC. 
Table 3 shows the combinations of errors in measurements of $\mathrm{Z}$ and LWP considered in the retrieval, and the errors are calculated for retrieved LWC with reference to true LWC. Cases 3 and 4 in table 3 are indicating that the retrieval is more sensitive to errors in LWP as compared to errors in Z with approximately the same mean absolute percentage error in LWC of $7 \%$ whatever the assumed errors in Z. This is because for each profile there is only one LWP value which impacts the whole profile for given error but for error in reflectivity, only the associated pixel is impacted. With the increase in percentage errors in LWP measurement from 1 to $100 \%$, the RMSE in LWC is also increased approximately 100 times, further demonstrating the high sensitivity of the algorithm to the LWP.

(Delanoë and Hogan, 2008) likewise incorporates a $1 \mathrm{dBZ}$ uncertainty in the measurement of $\mathrm{Z}$ for ice cloud retrieval using $95 \mathrm{GHz}$ radar with lidar and microwave radiometer. However, error in LWP has very low difference in MAPE and RMSE when $1 \%$ to $10 \%$ error is considered. Therefore case 6 in table 3, is an optimum balance of observational error for Z and LWP. This combination of error in measurement is used in all the retrieval cases presented in section 3.5 and 5.1 .

Table 3. Different configurations of error in measurement and respective statistical errors in retrieved LWC w.r.t. true LWC

\begin{tabular}{cccccc}
\hline Case & Error in Z & Error in LWP & RMSE(LWC) & $R^{2}(L W C)$ & MAPE(LWC)(\%) \\
\hline 1. & $1 \%(0.043 \mathrm{~dB})$ & $1 \%\left(1.01 \mathrm{gm}^{-2}\right)$ & 0.000209 & 0.99999 & 0.05783 \\
2. & $100 \%(4.34 \mathrm{~dB})$ & $1 \%\left(1.01 \mathrm{gm}^{-2}\right)$ & 0.000245 & 0.99999 & 0.15286 \\
3. & $1 \%(0.043 \mathrm{~dB})$ & $100 \%\left(2.71 \mathrm{gm}^{-2}\right)$ & 0.021870 & 0.98495 & 7.37329 \\
4. & $100 \%(4.34 \mathrm{~dB})$ & $100 \%\left(2.71 \mathrm{gm}^{-2}\right)$ & 0.021832 & 0.98499 & 7.43851 \\
5. & $25 \%(1.08 \mathrm{~dB})$ & $50 \%\left(1.64 \mathrm{gm}^{-2}\right)$ & 0.006013 & 0.99874 & 2.05276 \\
6. & $25 \%(1.08 \mathrm{~dB})$ & $10 \%\left(1.1 \mathrm{gm}^{-2}\right)$ & 0.000454 & 0.99999 & 0.17123 \\
\hline
\end{tabular}

\subsection{Sensitivity analysis of impact of attenuation due to liquid droplets model}

In this section, the sensitivity of the attenuation model considered in the algorithm to retrieve LWC is highlighted. Wet radome can cause up to $20 \mathrm{dBZ}$ of two-way attenuation due to rain in the reflectivity (Delanoë et al., 2016), but attenuation due to fog is far less than $20 \mathrm{dBZ}$. Two attenuation relations for liquid clouds from literature are used to test the sensitivity of the algorithm. Equation (10) is proposed by Vali and Haimov (2001) in which attenuation is a function of LWC (abbreviated as att(LWC) in table 4) and the relationship in Eq. (11) is proposed by Vivekanandan et al. (2020) where attenuation is the function of radar reflectivity factor (abbreviated as att(Z) in table 4). Both of these relationships are derived using in-situ observation from 95 $\mathrm{GHz}$ radar mounted on a research aircraft. Forward model with different attenuation relationships in the algorithm is tested for synthetic Z and LWC. To fabricate synthetic Z, the power law relation with $a=0.012$ and $b=2$ (in Eq. (1)) is used. Different combinations of attenuation correction in synthetic profile and in the retrieval algorithm are tested, as shown in table 4. $a$ priori for state parameters is calculated from Atlas (1954) empirical relation with error in a priori as $1000 \%$ and the measurement 
errors for Z and LWP are considered $25 \%$ and $10 \%$ as discussed in section 4.2. Finally, the comparison of bias in LWC for attenuation model is shown in figure 5 .

Table 4. Variation in error in a priori and different errors calculated w.r.t. true LWC

\begin{tabular}{ccccc}
\hline $\begin{array}{c}\text { Attenuation correction } \\
\text { in synthetic profile }\end{array}$ & Forward model attenuation & RMSE(LWC) & $R^{2}$ (LWC) & MAPE(LWC)\% \\
\hline Z (attLWC) & Att (LWC) & 0.000204 & 0.999998 & 0.056426 \\
Z (attLWC) & Att (Z) & 0.008286 & 0.997535 & 2.780574 \\
Z (attZ) & Att (LWC) & 0.008012 & 0.997687 & 2.660039 \\
Z (attZ) & Att (Z) & 0.000206 & 0.999998 & 0.057094 \\
\hline
\end{tabular}
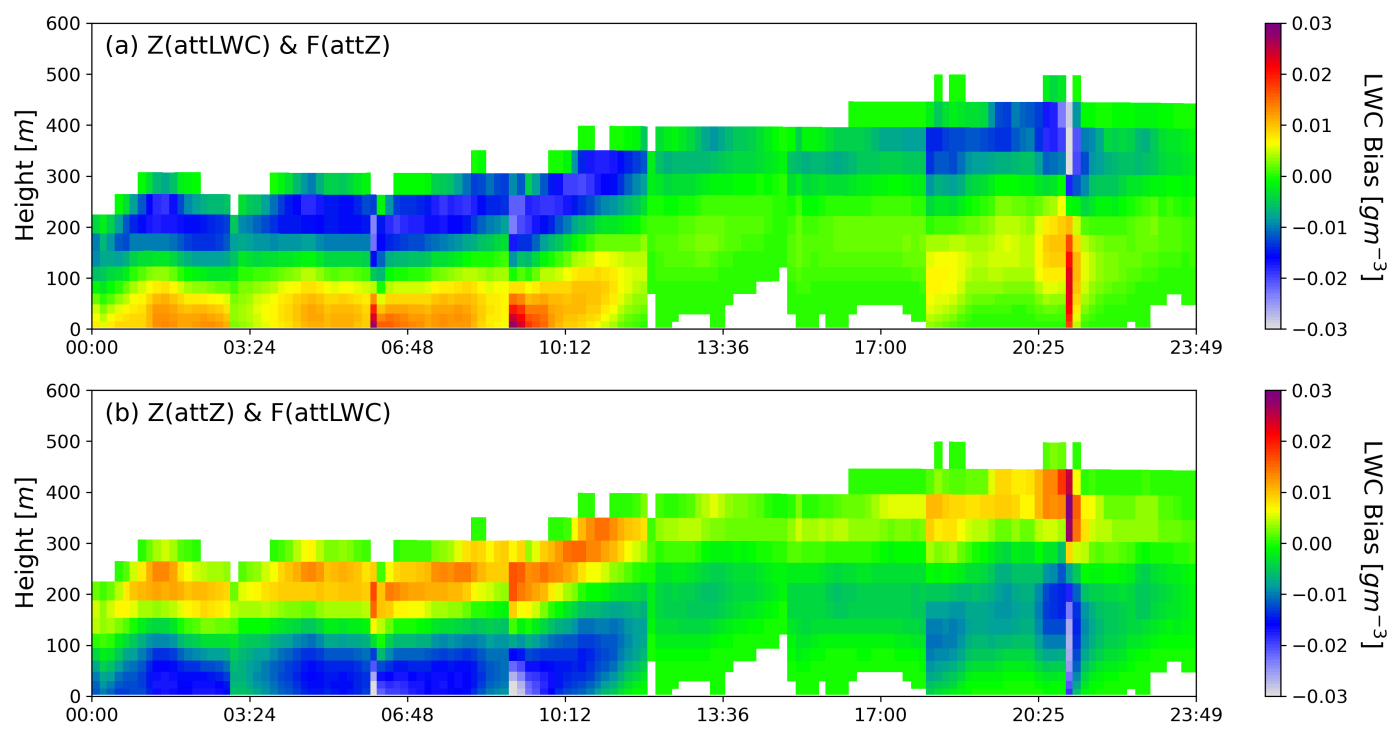

Figure 5. Bias in retrieved LWC with respect to true LWC for different attenuation consideration in the retrieval algorithm

Retrieved LWC considering same attenuation correction in synthetic Z profile and in forward model, RMSE is 0.0002 and MAPE is as low as $0.05 \%$ as all the parameters are identical. But when the attenuation relation is exchanged for synthetic profile and the forward model, MAPE increase to $2.7 \%$. Figure 5 shows the bias in LWC when different attenuation relation is used in the forward model and synthetic profile. The distribution of bias in LWC over the profile is different because attenuation due to LWC estimated by two relation is different, and thus the estimated LWC is also different. A similar test for attenuation with different ' $a$ ' in the power law relation gives the same errors when the retrieved LWC is compared with true LWC. Bias in LWC for considering same attenuation relation in synthetic profile and forward model is found close to zero. Therefore, the 
sensitivity test for attenuation indicates that attenuation correction of $Z$ has very low-impact, and it can bring up to $2.7 \%$ mean absolute percentage error in retrieved LWC when wrong attenuation model is used.

\subsection{Sensitivity analysis of bias in $\mathrm{Z}$ and LWP}

Bias in observation is the systematic error added in measurement, which can be due to the error in calibration of any instrument or transfer function of the measurement. Similarly, threshold value of MWR also adds a systematic error in LWP measurement. Therefore, it is necessary to test the behavior of retrieval algorithm for such systematic biases in measurement. For the test cases of biases, error in observation vector in considered $25 \%$ and $10 \%$ for Z and LWP with a priori of LWC is calculated using $a=0.012$ proposed in Fox and Illingworth (1997) and $a=0.012$ is used as lna a priori. This test is to analyze the impact of bias in measurement on retrieval, therefore the a priori should have minimum contribution and hence $1000 \%$ error in a priori of LWC and lna is considered. In this analysis, only one of the two observations is biased at a time to see the individual impact on retrieval. It is assumed that the bias in $\mathrm{Z}$ is $2 d B Z$ considering that error in calibration in BASTA radar measurements is around 1 to $2 d B Z$ (Toledo et al., 2020). The bias in LWP estimation is considered $10 \mathrm{gm}^{-2}$ which is supported by Wærsted et al. (2017) for this sensitivity test.

Table 5. Error in retrieved LWC due to bias in Z and LWP

\begin{tabular}{ccccc}
\hline Case & Bias & RMSE(LWC) & $R^{2}(\mathrm{LWC})$ & MAPE(LWC)\% \\
\hline 1. & LWP-10 $\left(\mathrm{gm}^{-2}\right)$ & 0.029413 & 0.96343 & 11.246633 \\
2. & LWP+10 $\left(\mathrm{gm}^{-2}\right)$ & 0.030236 & 0.97184 & 11.542570 \\
3. & Z-2 (dBZ) & 0.000355 & 0.99999 & 0.131603 \\
4. & Z+2 (dBZ) & 0.000558 & 0.99998 & 0.210887 \\
\hline
\end{tabular}

The order of error in retrieved LWC with respect to true LWC is much higher for $10 \mathrm{gm}^{-2}$ bias in LWP than $2 d B Z$ bias in $\mathrm{Z}$. However, the bias in two measurements is not comparable because parameter $\mathrm{Z}$ is measured over each pixel and LWP is a single point measurement for the whole column. Since the bias applied on $\mathrm{Z}$ applies on each cloud pixel and bias applied in LWP is integrated for whole profile, however, $11 \%$ MAPE in LWC is observed which is again indicating the sensitivity of retrieval for LWP. Another reason for the difference in LWC is due to the fact that $\mathrm{Z}$ is in $\log$ space and error in observation allows more spread in Z (25\%) than in LWP (10\%) therefore the impact on LWP is larger. The bias in Z is propagated in lna, but the bias in LWP directly impacts LWC. The simultaneous biases in Z and LWP have been also tested, which reveals that the bias in LWP is dominating over the bias in $\mathrm{Z}$ with $11 \%$ MAPE when mentioned biases are considered in $\mathrm{Z}$ and LWP.

\subsection{Sensitivity of parameter $b$}

500 The exponent $b$ from the power law Eq. (1) is considered 2 for all the cases discussed in this paper, however the range of parameter $\mathrm{b}$ in the literature is proposed from 1 to 2 . To test the impact of variation in $b$ on the retrieval algorithm, the value 
of $b$ used to fabricate synthetic observations $\mathrm{Z}$ and LWP, and $b$ in the forward model are same. Keeping all the other settings constant, the error in retrieved LWC should be due to changing $b$. Table 6 , shows the range of $\mathrm{b}$ and the respective error in retrieved LWC with respect to true LWC. The retrieved LWP matches with the assimilated LWP only the distribution of LWC is changed observed least for $b=2$. Figure 6 shows that the cost function is also least for $b=2$ and MAPE in LWC is twice when the value of $b$ is taken 1 .

There is negligible impact of variation in $b$ over $\ln a$ as shown in figure and the error in LWC is between $0.35 \%$ to $0.17 \%$. The convergence is achieved with less cost function and MAPE in LWC is also least for $b=2$ case. Because lna is allowed to be variable in the forward model, it is most likely that the change in $b$ is compensated by the change in lna.

\subsection{Analysis of the sensitivity exercise}

In conclusion, since this sensitivity test was performed on a synthetic profile, the overall impact of uncertainty of each parameter on the retrieval can be very different when applied to a real profile. However, an estimate of errors can be made using this exercise. The error in observation must be chosen very carefully for retrievals. $25 \%$ error in $\mathrm{Z}$ is also supported by realistic calibration error of BASTA radar which was calculated between 1 and $2 d B Z$ using $20 \mathrm{~m}$ mast (Toledo et al., 2020) where $25 \%$ error in $\mathrm{Z}$ corresponds to $1.08 \mathrm{dBZ}$. This combination of $25 \%$ and $10 \%$ error in measurement has only $0.17 \%$ MAPE when tested with synthetic profile, which is why this combination is used in the algorithm. a priori must be considered only to stabilize the retrievals for unavailable measurements, otherwise the error in a priori can be kept high. A prior is a constraint for the entire retrieval, hence the uncertainty in the retrieval must be smaller than the error in a priori. Otherwise, the retrieval does not add any information from the observations (Maahn et al., 2020). Attenuation by liquid cloud droplets is yet unknown for continental cloud however the available relations from literature proposed for marine clouds is used in the retrievals might bring up to $2.7 \%$ error in retrieved LWC. Retrieval is very sensitive to bias in LWP as LWP is point information for whole cloud column, therefore error in observation and biases in $\mathrm{Z}$ and LWP both play a very critical role in the retrieval. Sensitivity of retrievals for parameter $\mathrm{b}$ is showing the least error when $b=2$ because this is the same used to fabricate $\mathrm{Z}$ synthetic from the true LWC. Nevertheless, it is worth noting even with other values of $b$ the MAPE is not exceeding $0.35 \%$.

Table 6. Error in retrieved LWC for different $b$ values

\begin{tabular}{ccccc}
\hline Case & $b$ value & RMSE (LWC) & $R^{2}(L W C)$ & MAPE (LWC) \\
\hline 1. & $\mathrm{~b}=1$ & 0.00069 & 0.99998 & 0.35599 \\
2. & $\mathrm{~b}=1.2$ & 0.00064 & 0.99998 & 0.301158 \\
3. & $\mathrm{~b}=1.4$ & 0.00059 & 0.99998 & 0.260569 \\
4. & $\mathrm{~b}=1.6$ & 0.00054 & 0.99998 & 0.227267 \\
5. & $\mathrm{~b}=1.8$ & 0.00050 & 0.99999 & 0.198041 \\
6. & $\mathrm{~b}=2$ & 0.00045 & 0.99999 & 0.171237 \\
\hline
\end{tabular}



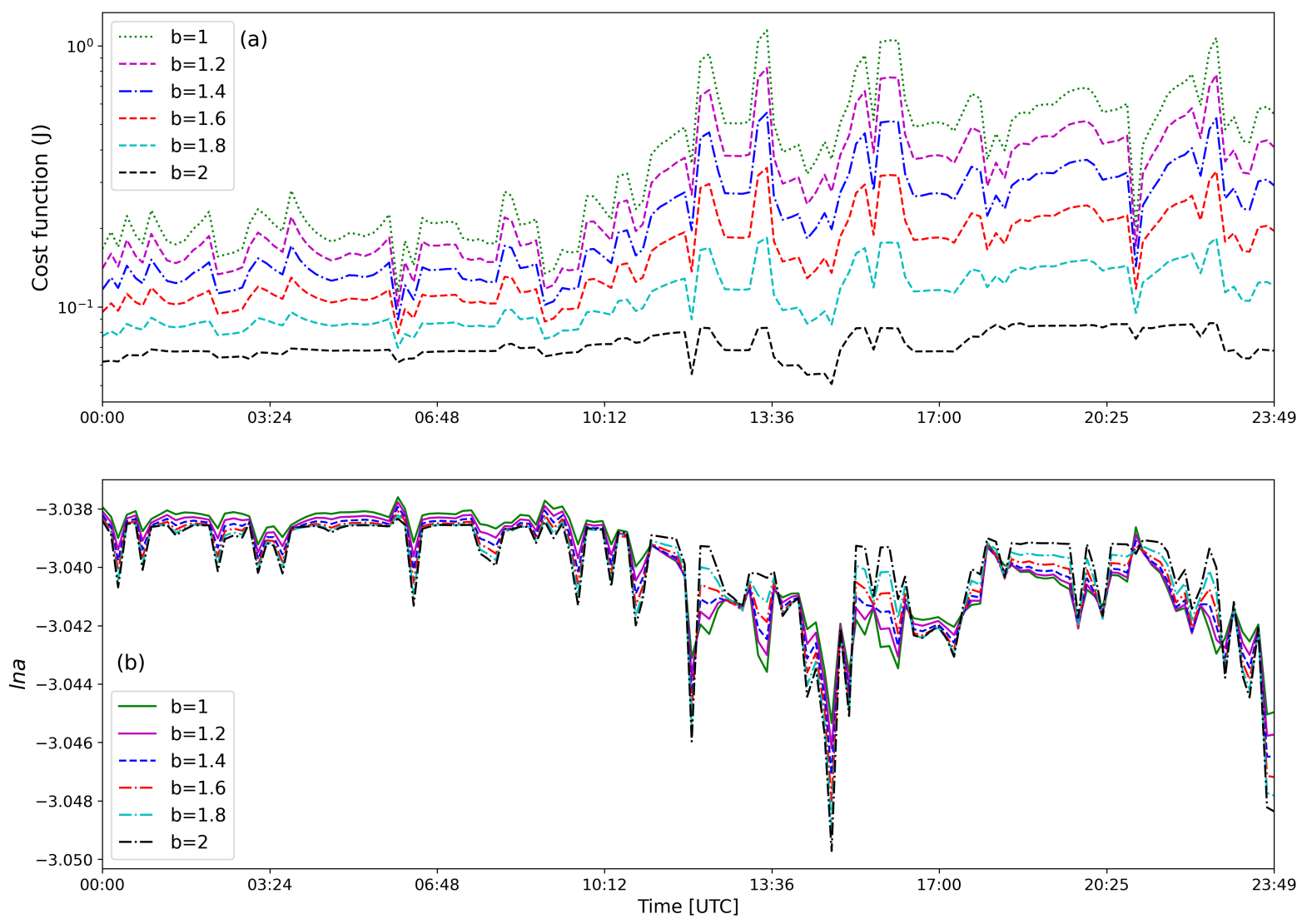

Figure 6. (a) Cost function and (b) Retrieved lna for different $b$ values

\section{Comparison of $L W C$ retrieval with in-situ data}

In-situ measurements of cloud and fog are required to validate the distribution of LWC with time and height. In general, in-situ measurements of cloud microphysical parameters are collected using a research aircraft mounted with sensors flying inside the cloud. During the SOFOG-3D field experiment, a tethered balloon equipped with in-situ sensors was used to collect the microphysical parameters of fog. This approach is much more economical than the research aircraft flying inside cloud, however the trajectory of the balloon cannot be fully controlled, and the measurements are limited to the lowermost 1-2 $\mathrm{km}$ level. Simultaneous measurements using remote sensing instruments like BASTA cloud radar, microwave radiometer and automatic weather stations were also collected for various fog cases (Martinet et al., 2020). Since the LWC retrieval algorithm described in previous sections essentially works with liquid clouds including fog, measurements collected during the SOFOG3D experiment are well suited to validate the algorithm. The input for the algorithm is taken from vertically pointing cloud radar reflectivity and LWP estimates from MWR measurements. Retrieved LWCs are then compared with the measured LWC using in-situ sensors. 


\subsection{Presentation of the case study of 09 February 2020}

One fog case study observed at the super-site $\left(44.4^{\circ} \mathrm{N},-0.6^{\circ} \mathrm{E}\right)$ on 9 th February 2020 is presented to compare retrieved LWC with in-situ measurements collected from the tethered balloon. This case is selected because fog and stratus were observed, allowing us to validate the algorithm for two different cloud types at once. The observations from vertically pointing radar and MWR are used to retrieve LWC with exactly the same algorithm described in previous sections. During this experiment, MWR was set up to collect boundary layer scan at lower elevation angle down to $4^{\circ}$ every 10 minutes and therefore the LWP is interpolated for such gaps. Relying on the previously led sensitivity study, error in observations for Z and LWP is taken as $25 \%$ and $10 \%$ respectively, with a priori information calculated from Atlas (1954) empirical relation. Error in a priori is considered $1000 \%$ which is the same as mentioned in section 3.3 when MWR information is available. As stated in section 3.3, radar misses a few low level gates near the ground due to antenna coupling, which contains critical fog information. The properties of fog are assumed to remain constant between the first available gates and the ground, and thus reflectivity is extrapolated (extended) downwards for the unavailable range gates. The fog shown in figure 7 sustained for 4 hours and then started dissipating to form a stratus cloud. Figure 7(a) illustrates radar reflectivity extended to the lowest gates, whereas in figure 7 (b) Doppler velocity is plotted only for the available gates. Higher velocity at the fog top are indicating the entrainment process causing the dissipation of fog after 04:00 hours. The visibility observed at the super-site is also less than $1000 \mathrm{~m}$ until 04:00 hours. The discontinuity in radar reflectivity close to $200 \mathrm{~m}$ is due to the beam overlap correction used in L2 product of BASTA.

The distribution of retrieved LWC over time and height is shown in figure 7(c) along with the trajectory of the tethered balloon. Figure 7(d) and (e) are the plots for LWP and retrieved lna respectively for this case.

\subsection{Comparison between in-situ and radar measurements}

To compare the retrieved LWC with in-situ measurement, the co-location of tethered balloon data with BASTA reflectivity points is accomplished by determining the closest radar gate that corresponds to the balloon height.

In figure 8(b) and (c) the black dashed line indicates that the visibility is more than $1000 \mathrm{~m}$ from 04:00 hours onwards and therefore separates fog and stratus cloud. Since the balloon also contaminates the radar measurement, therefore all the colocated points when the tethered balloon was within the radar detection range are eliminated. The maximum distance observed between the tethered balloon and BASTA radar was $700 \mathrm{~m}$. Radar reflectivity factor from in-situ measurements is calculated using the 6th moment of the droplet distribution measured by CDP. Note that the radar reflectivity is still in the Rayleigh regime as the measurements from CDP cannot exceed $50 \mu \mathrm{m}$. The co-located points with reflectivity less than $-40 \mathrm{dBZ}$ are masked because the signal-to-noise ratio for radar is low.

In figure 8(b) the radar reflectivity from BASTA and CDP are compared for the co-located points and indicates a clear bias for fog and relatively much better agreement for stratus cloud with $-4.44 \mathrm{dBZ}$ mean bias for fog and $0.89 \mathrm{dBZ}$ for stratus cloud. The bias is calculated as the difference between $Z_{B A S T A}$ and $Z_{\text {in-situ }}$. The root mean square error (RMSE) in $\mathrm{Z}$ is 5.2 $d B Z$ for fog and $2.8 d B Z$ for stratus. Figure 8 (c) shows the comparison of the retrieved LWC values with LWC observed by 

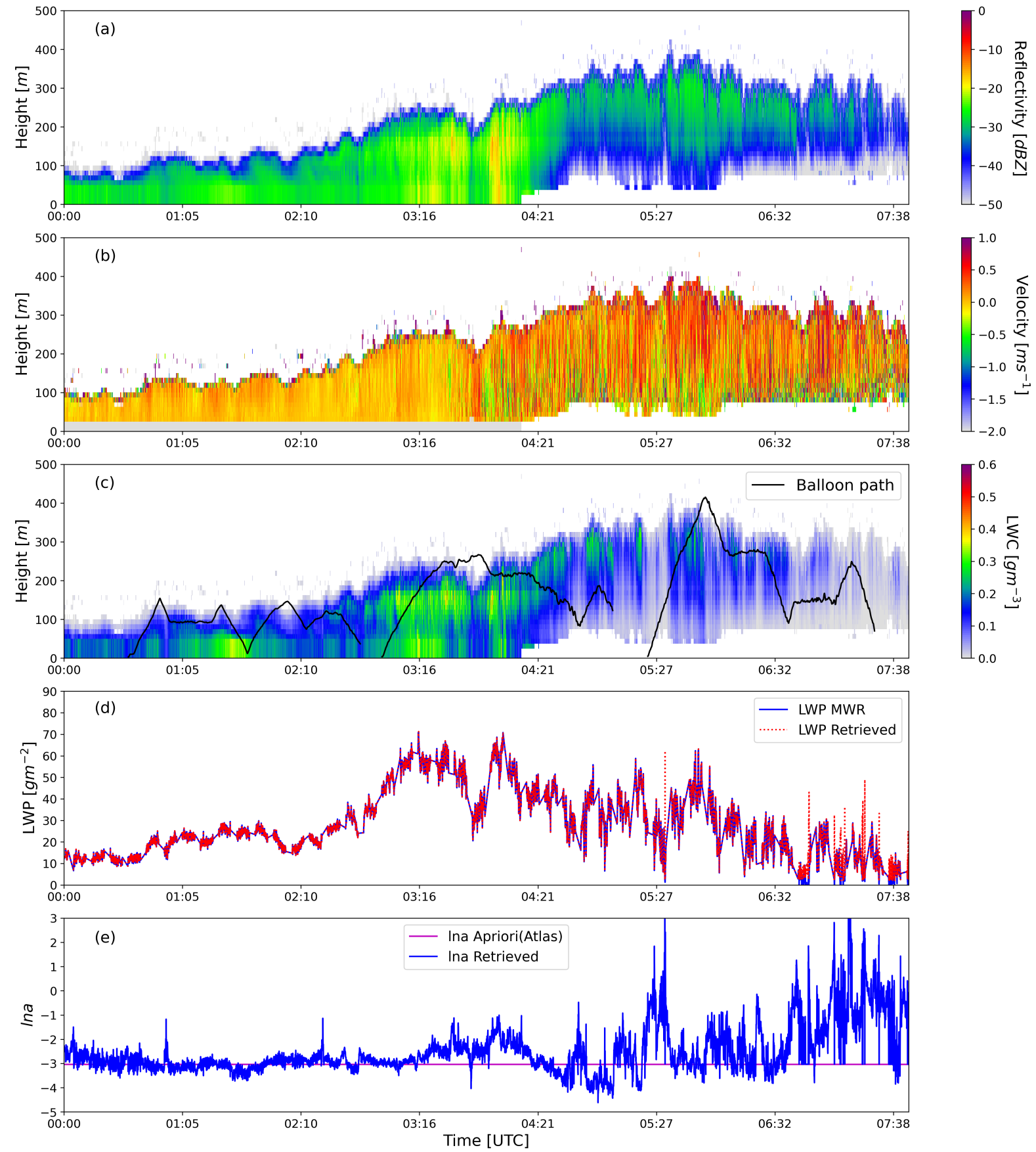

Figure 7. (a) Radar reflectivity Z (b) Vertical velocity (c) Retrieved LWC, (d) LWP and (e) Retrieved lna for 09 February 2020 case at SOFOG-3D super-site. Tethered balloon trajectory over retrieved LWC is shown in black line. 
https://doi.org/10.5194/amt-2022-3

Preprint. Discussion started: 31 January 2022

(c) Author(s) 2022. CC BY 4.0 License.
Atmospheric

Measurement

Techniques

Discussions
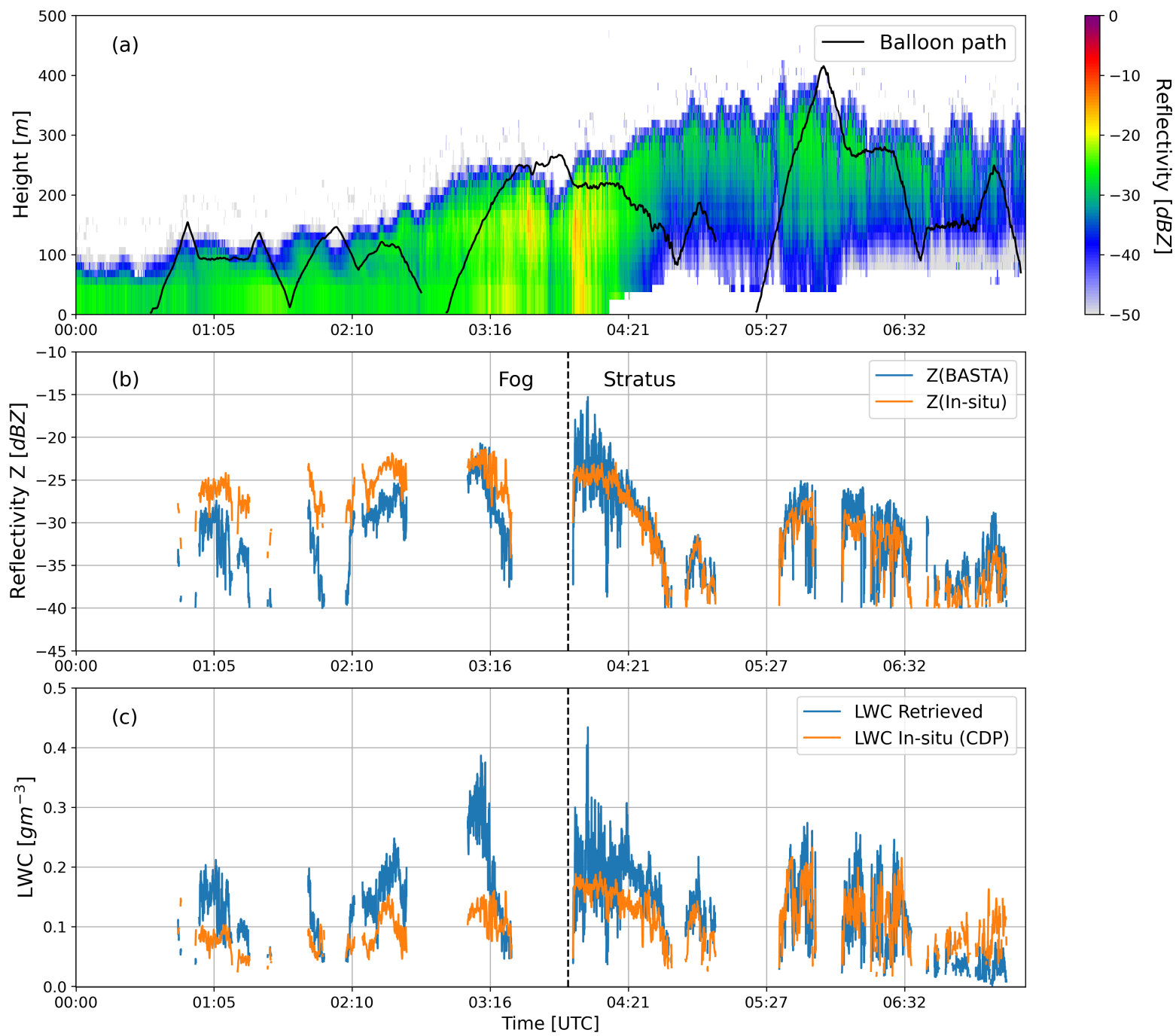

Figure 8. (a) Radar reflectivity and balloon path (b) Comparison of radar reflectivity with reflectivity calculated from CDP using DSD (c) Comparison of retrieved LWC with in-situ LWC 
$570 \mathrm{CDP}$ at the co-located points of the balloon trajectory. The mean bias in LWC for fog is $0.06 \mathrm{gm}^{-3}$ and for stratus cloud is $0.009 \mathrm{gm}^{-3}$. The root mean square error (RMSE) in LWC for fog is $0.082 \mathrm{gm}^{-3}$ and $0.056 \mathrm{gm}^{-3}$ for stratus. The comparison of retrieved LWC with in-situ observations of LWC from CDP resulted in a root-mean-square error of $0.067 \mathrm{gm}^{-3}$ including fog and stratus.

For a well-calibrated radar, the reflectivity estimated from in-situ sampling should match with the radar reflectivity if both the instruments are sampling the same cloud column and have a similar sensitivity to DSDs. The sensitivity of the CDP sensor is limited to sample the droplet diameters from 2 to $50 \mu \mathrm{m}$, while radar can sample a wider range of DSDs and is more sensitive to the largest droplets. The variations in comparison with in-situ observations are noticed when the balloon is close to the cloud edge, where a slight difference in altitude can have a significant impact on $\mathrm{Z}$ and LWC due to the heterogeneity of this area.

The observed differences in simulated $\mathrm{Z}$ and radar measurements could be explained by the vertical and horizontal heterogeneity of the fog, which strongly depends on the fog maturity. To further investigate the fog stages, a broader perspective beyond the vertical profile of fog is required. Multiple remote sensing and in-situ instruments were operated simultaneously as part of the SOFOG-3D campaign to explore various fog properties. A $95 \mathrm{GHz}$ scanning radar called BASTA-mini has been centered $1 \mathrm{~km}$ away from the vertically pointing radar, and the $360^{\circ}$ scan of fog is presented in figure $9(\mathrm{a})$ and (b). Plane Polarised Indicator (PPI) of scanning radar shown in figure $9 a$ and $9 b$, are collected at $4{ }^{\circ}$ elevation angle. Note that this low elevation of radar can also be contaminated by the ground clutter, indicating locally high reflectivity. In the figure $9 \mathrm{~b}$, a larger spread of fog is observed, which is due to the development of thicker fog.

Due to the constant evolution of fog stages and the horizontal heterogeneity of fog, the sampled volume away from the vertically pointing radar will also have distinct $Z$ and LWC. As shown in figure $9 \mathrm{~b}$, the distribution of reflectivity in the left and right-hand side of scanning radar is different. Therefore, the mismatch in $\mathrm{Z}$ and LWC can be explained by different radar and CDP sampling volumes. As the fog lifted into stratus cloud around 04:00 hours, we can observe a better agreement in figure 8(b) and (c), which could be explained by a more homogeneous situation. Furthermore, as shown in figure 8 (a), samples are not collected at the cloud edge for stratus and therefore have lesser uncertainties in $\mathrm{Z}$ and LWC.

In order to have a better idea of the representativeness of CDP in-situ data, a scatter plot of retrieved LWC with radar reflectivity from BASTA radar and in-situ measurement of LWC with simulated radar reflectivity using DSD compared with empirical Z-LWC relationships in figure 10. In-situ measurements are separated for fog and stratus clouds where magenta colour denotes fog, yellow-green (Chartreuse) colour denotes the stratus cloud, and the respective linear fits are also plotted. Various Z-LWC relations for clouds are included in table1, but are not proposed for fog. In Dupont et al. (2018), linear fits for fog are proposed based on in-situ observations from the tethered balloon and BASTA cloud radar at SIRTA. As a reference for fog, Flight1, Flight2, and Flight3 in the figure 10 are the fits for three fog instances computed by relating LWC observations from a light optical aerosol counter (LOAC) sensor to BASTA measurements, as described in Dupont et al. (2018). These Z-LWC fits for fog are obtained by finding the linear fit of LWC from LOAC sensor to the radar reflectivity $\mathrm{Z}$ of the closest gate from vertically pointing BASTA radar. We compared the behaviour of in-situ fog measurement during the SOFOG-3D campaign to that of other fog relationships. As illustrated in figure 10, no empirical relation from the literature, including the one derived in fog, seems to be able to represent the in-situ observations of this fog situation. However, the scatter for in-situ 


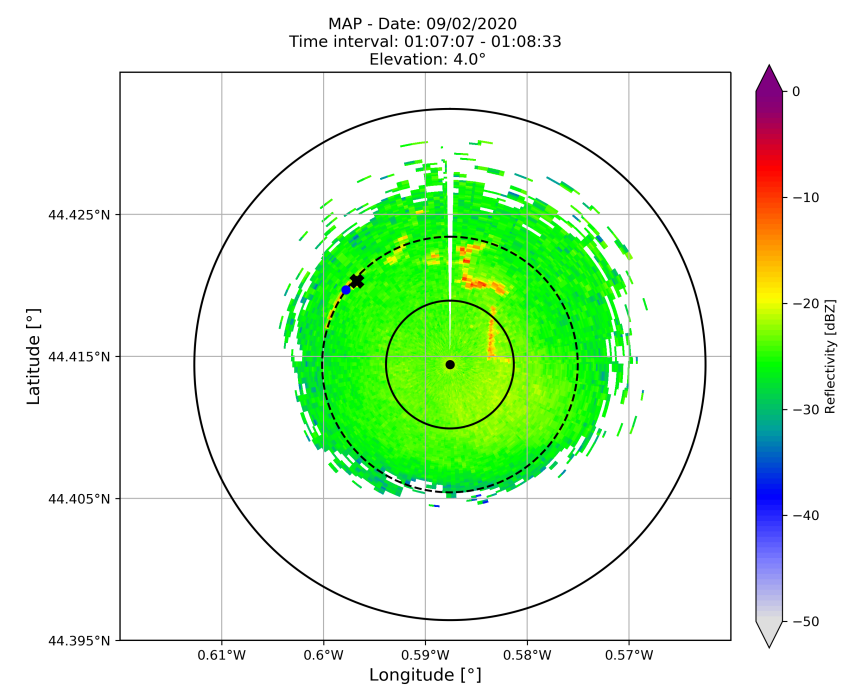

(a) Scan at 01:07 hours

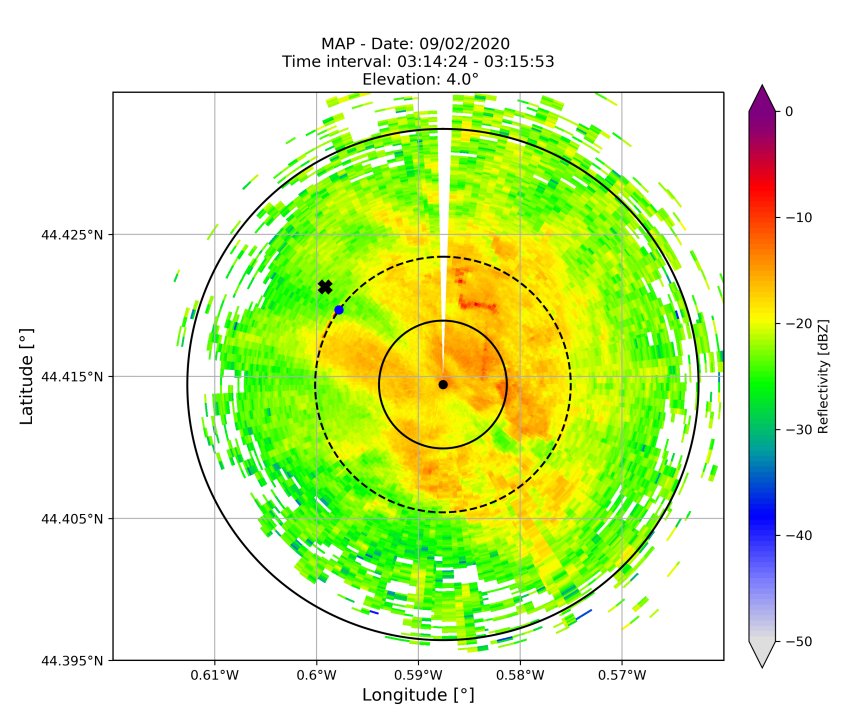

(b) Scan at 03:14 hours

Figure 9. Scans of BASTA-mini collected for fog at $4^{\circ}$ elevation angle. The vertically pointing radar shown as blue dot was located $1 \mathrm{~km}$ away from the scanning radar and cross represents the location of balloon.

measurements of stratus represents a good correlation with other empirical relations as well as with the linear fits for fog from Dupont et al. (2018). The in-situ measurements separated for fog and stratus clearly show different characteristics and also indicate that different reflectivity values for the same LWC can be obtained as shown in figure 10a. This could be because of the diverse droplet spectra in stratus and fog.

The impact of various DSD characteristics during the fog stages in the simulation of different radiation fogs is discussed in Maier et al. (2012). In the Raleigh regime $\mathrm{Z}$ values might get larger as fog develops due to the increase in droplet radius, while the LWC may remain constant. This introduces a non-linear relation between LWC and radar reflectivity Z. The variability within each fog stage exhibited unique properties depending on the fog event (Maier et al., 2012).

In figure 10b the retrieved LWC from the algorithm with respect to BASTA reflectivity is plotted in blue scatter, and it matches only with the in-situ observations for stratus and other empirical Z-LWC relations. In-situ fog indicates relatively less LWC than stratus cloud at the same radar reflectivity. For the sake of comparison with Dupont et al. (2018), we also related the in-situ LWC obtained during SOFOG-3D with co-located radar reflectivity from BASTA. By correlating in-situ measurements of LWC with cloud radar reflectivity, it is assumed that the radar and in-situ sensor are observing the same cloud volume; however, distance between the balloon and the nearest gate of cloud radar can incorporate uncertainties. In addition to this, the sensitivity of the in-situ sensor (CDP) and radar (BASTA) is considered the same, despite the fact that the sensitivity 
varies with DSDs. Generally, the cloud probes under sample the true DSD of the volume due to their limited sensitivity to droplets.As shown in figure 11, the Z-LWC fits from in-situ observation are in neighborhood to other empirical relation for reflectivity less than $-30 d B Z$. Since the power-law relations are valid only in the Rayleigh regime, the in-situ observation agrees with other empirical relations for low reflectivity. Reflectivity values greater than $-30 d B Z$ may be attributed to larger droplets, which may or may not include a higher LWC. However, a significantly better correlation of in-situ fit for stratus cloud with empirical relation by Baedi et al. (2000) (proposed for stratocumulus clouds) indicates representativeness of in-situ observations for stratus. The fit for in-situ fog observation still indicates less LWC at the same reflectivity and does not match with any empirical relation. These observations imply that these are either collected for large droplets beyond the CDP limit or from a sampling volume distinct from the one cloud radar samples.

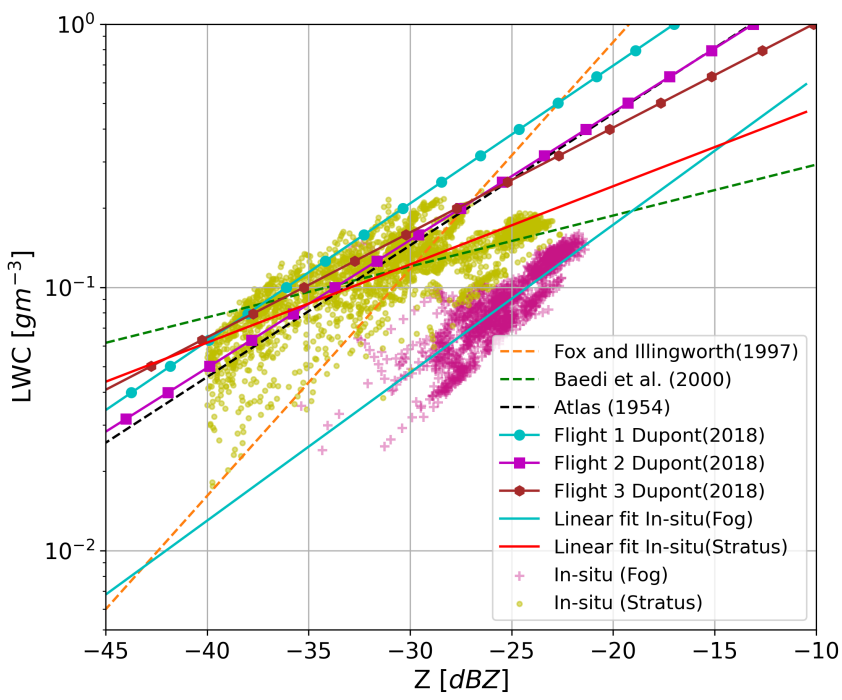

(a)

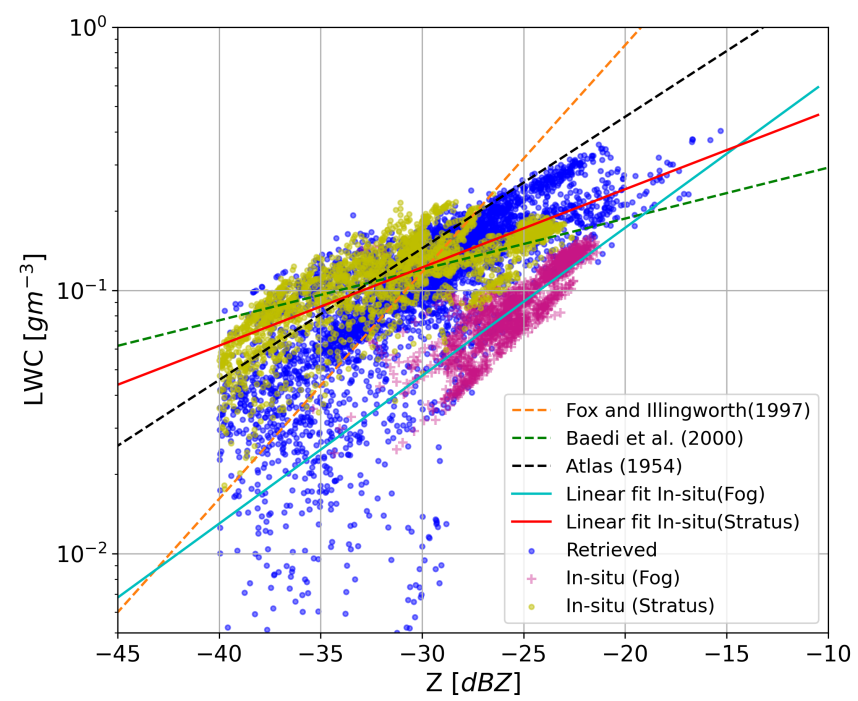

(b)

Figure 10. Comparison of in-situ LWC and radar reflectivity relation with (a) available literature for fog and clouds, (b) retrieved LWC and BASTA radar reflectivity relation.

Unfortunately, the limited in-situ observations collected for fog and stratus here do not represent a validation of the retrieval; however, this comparison highlights that there are situations more complicated than the other. Due to the non-uniform distribution of LWC in cloud or fog, homogeneity plays a key role while validating with the in-situ measurements. It is unfair to expect LWC to match when simulated reflectivity from in-situ does not match radar measurement. In order to validate such an algorithm, in-situ measurements at different heights for the same volume that radar samples are needed. However, if the in-situ observation platform is positioned in proximity to the radar sampling volume, it may also contaminate the radar observations. Therefore, the in-situ measurements must be collected from a homogeneous cloud to compare with the retrievals. Particularly for fog, more continuous DSD measurements as well as the vertical profiles during distinct fog episodes are required to produce more significant results. 


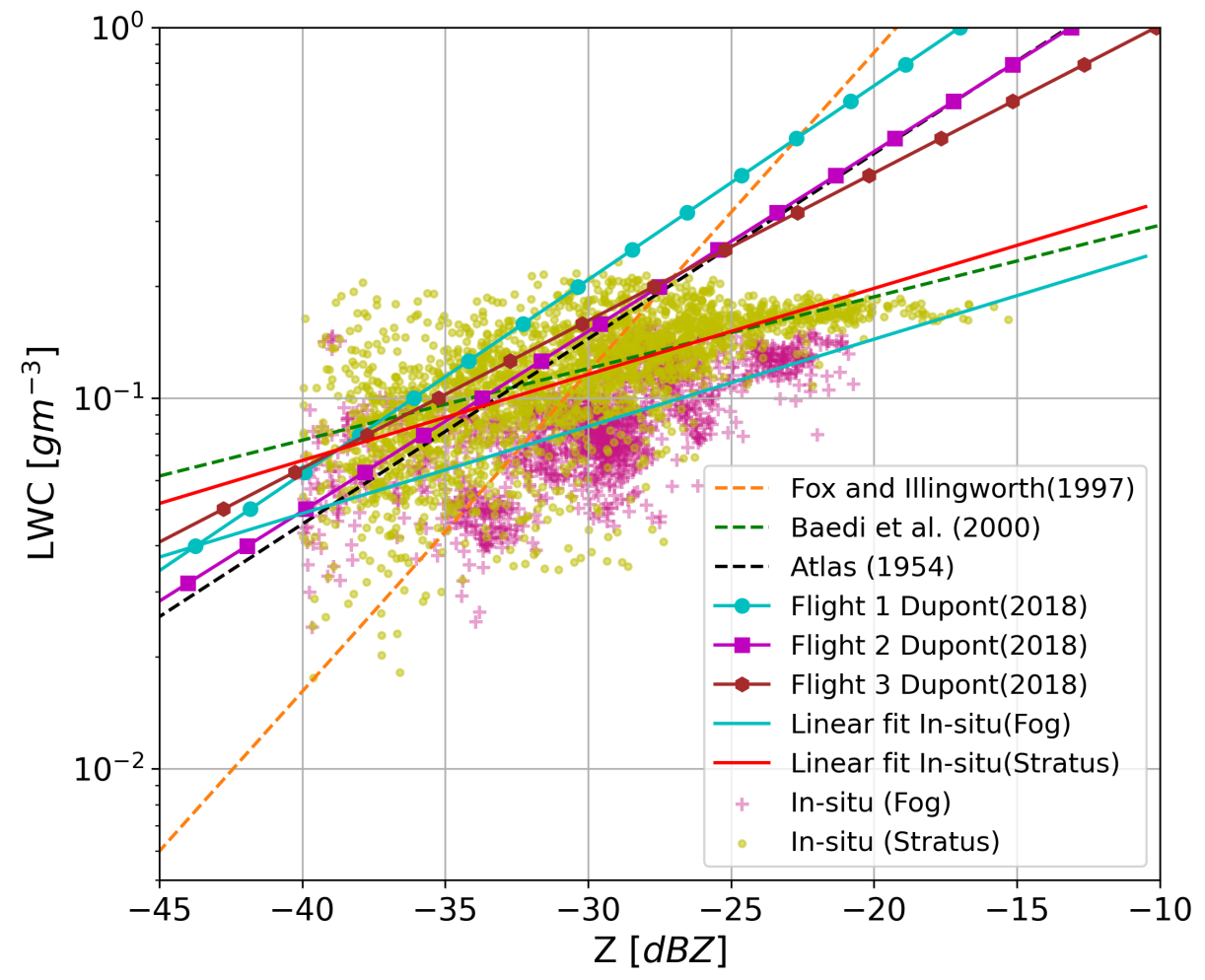

Figure 11. Scatter plot for relation between LWC measured from CDP with radar reflectivity from cloud radar, compared with available literature

\section{Statistical analysis of retrievals to derive climatology}

The primary objective of this statistical analysis is to derive a climatology of LWC and lna in order to allow the algorithm to be able to retrieve LWC for fog and low-level liquid clouds even when additional measurements are not available. As presented in section 5.1, when the retrieved LWC is compared with in-situ LWC measurements for fog and stratus cloud from the SOFOG$3 \mathrm{D}$ experiment. Therefore, the climatology is developed from the retrieval technique discussed in section 3.5 using the larger data set from SIRTA measurements for a variety of cloud and fog incidents. Statistical analysis to derive a climatology of LWC and scaling factor is presented in this section. Figure 12 presents the histogram of observed parameters followed by retrieved parameters for the selected observation set.

The histogram of the retrieved scaling factor lna indicates that, the highest values of occurrence are around - 3 which is close to the lna a priori value from (Atlas, 1954) the empirical relation plotted as the red line, but it is not precisely the same. The variational framework allows variability in the lna retrieval. The assimilation of LWP brings enough information to retrieve $\ln a$ and the spread around the a priori value is directly linked to the a priori error value. Table 1 indicates the $\ln a$ values for various cloud types proposed in the literature, which agree well with the range of retrieved lna values. Note that there is one 

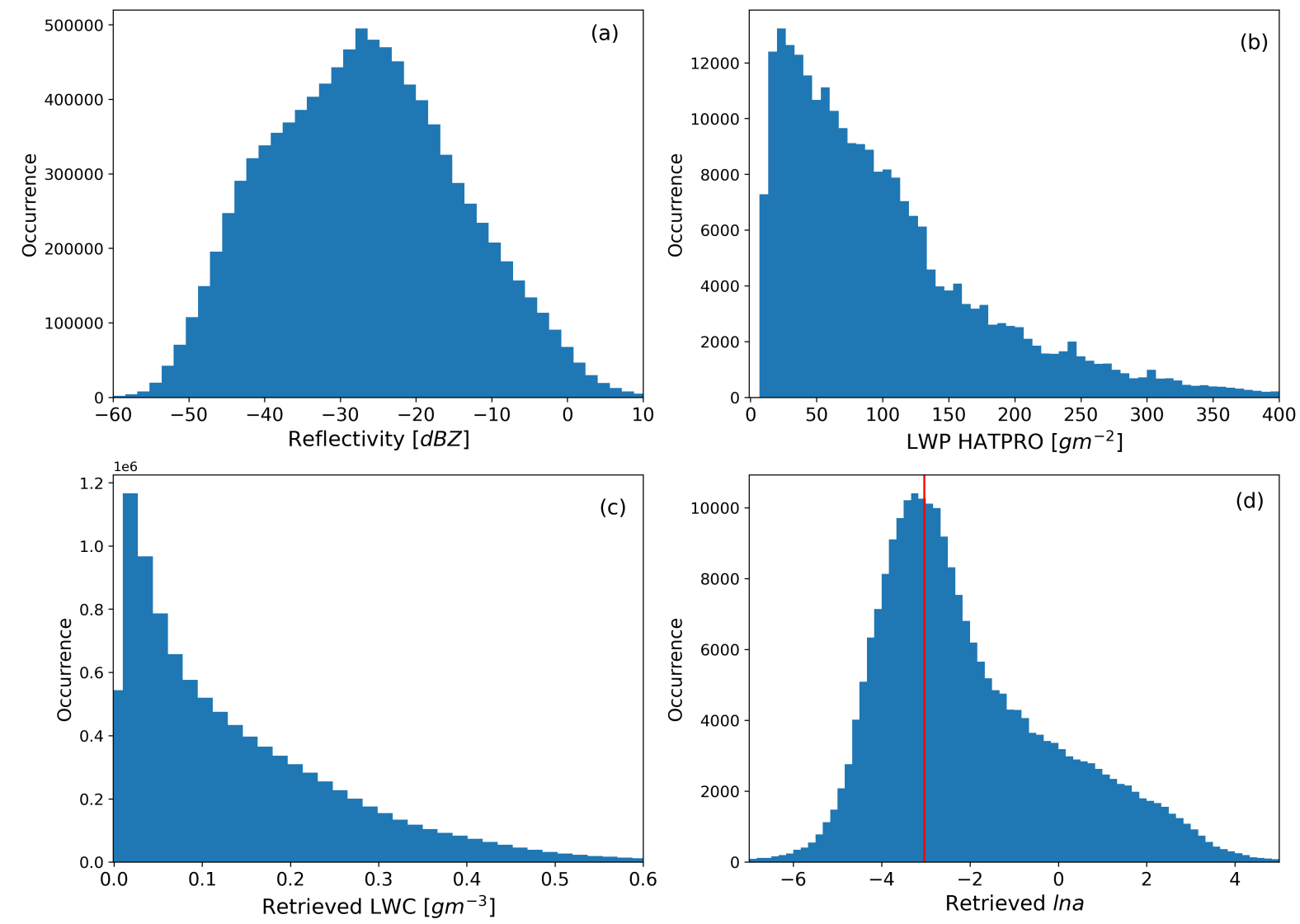

Figure 12. Histogram of (a) Radar reflectivity(Z) (b) LWP from MWR (c) Retrieved LWC (d) Retrieved lna for 39 cloudy days, and the red line in lna histogram indicates the a priori of lna from table 1

single lna value for a given profile, but its value can potentially be used to differentiate clouds from drizzle. All the profiles with rain and drizzle reaching ground are removed for the statistics, however light drizzle with clouds and fog is discussed.

Since the algorithm does not assimilate LWP for the profiles with LWP less than $10 \mathrm{gm}^{-2}$, LWP histogram in the figure 12(b) has no value below $10 \mathrm{gm}^{-2}$ and maximum cloud profiles has the LWP below $120 \mathrm{gm}^{-2}$.

The parameter LWC is indicating the range up to $0.6 \mathrm{gm}^{-3}$ which includes light drizzle, while the highest number of cloud pixels have LWC value less than $0.2 \mathrm{gm}^{-3}$. In figure 13, retrieved LWC is plotted as a function of radar reflectivity for the 39 cloud cases, with Z-LWC empirical relationships from literature for various cloud types. The black line represents a priori of the retrieval algorithm, and the higher concentration of density points overlaps with the black line is due to the profiles with $\mathrm{LWP}<10 \mathrm{gm}^{-2}$ where the retrieval of LWC is based on only Atlas empirical relation. All these profiles are not considered in the climatology of lna. However, the wide range retrieval points indicates that the algorithm allows LWC retrieval for a variety of cloud types. The slope of Z-LWC relationship is dependent on the value of $b$ in equation 9 and because the retrieval method 


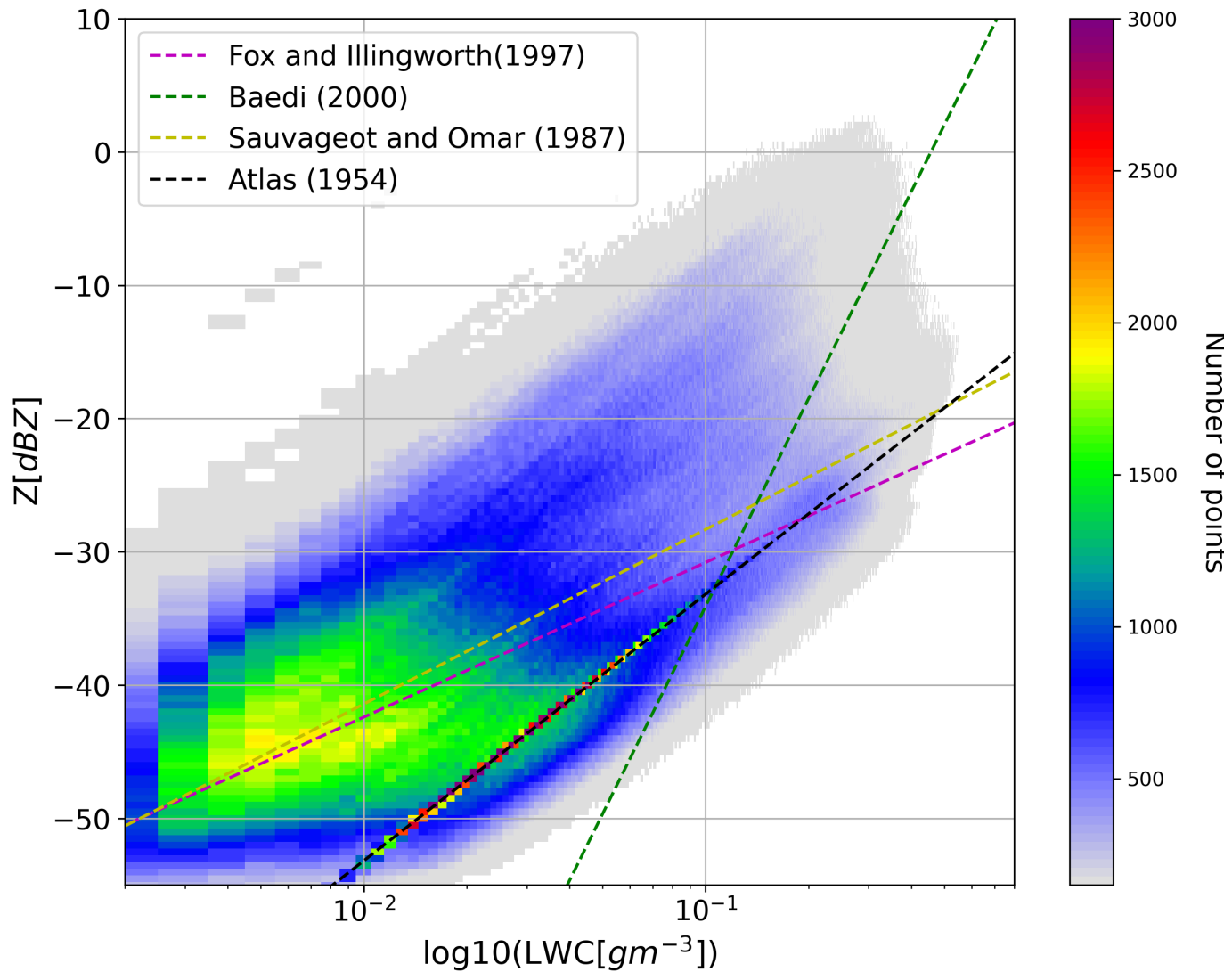

Figure 13. Retrieved LWC as a function of radar reflectivity $\mathrm{Z}$ for 39 cloudy days, with reference plot of various empirical relation for different cloud types.

considers $b=2$, the slope of the total retrieval in figure 13 is constant. However, retrieval allows variability in lna which could partly compensates for $b$ as well.

As already described, knowing LWP allows us to retrieve lna and adjust the relationship between LWC and Z. However, when only BASTA measurements are available, we need to rely on an a priori value for lna. Thanks to this climatology we could both define the optimal value for this a priori and also eventually propose to parametrise this value, for instance it is envisioned to relate the scaling factor to radar reflectivity and/or Doppler velocity. As Z and V are observed for each cloud pixel and only one value of lna is retrieved for a given cloud profile, one single reflectivity or velocity information should be associated with lna. We propose to summarise the reflectivity and velocity information to the mean or maximum value of the profile, in order to have one value per profile.

Maximum and minimum velocities of the cloud column are associated with the updrafts and downdrafts, which may not represent the complete profile for lna. Therefore, we rely on the mean velocity of the profile. The density plot of mean velocity as a function of lna is plotted in figure 14(a) indicating that mean velocity of most profiles is concentrated between -0.5 to 0.1 
$m s^{-1}$ which is compatible with liquid cloud sedimentation velocity. Mean velocity close to $0 \mathrm{~ms}^{-1}$ with $\ln a$ values ranging from -4 to -2 implies pure clouds, whereas few profiles with the mean velocity less than $-0.5 \mathrm{~ms}^{-1}$ must be impacted by the drizzle droplets in the profile. Standard deviation plotted in the red line indicates that the variability of lna is very high for the profiles with mean velocity below $-0.5 \mathrm{~ms}^{-1}$. Due to the large standard deviation, lna cannot be associated with mean velocity, however velocity information can be used to classify drizzle droplets. As illustrated in figure 14(b), a substantially stronger association is observed between maximum radar reflectivity and lna of the profile. For most of the cloud columns, maximum reflectivity is observed between -30 to $-15 d B Z$. As maximum reflectivity also represents the drizzle in cloud, the maximum reflectivity above $-10 d B Z$ is suspected to indicate drizzle in cloud. High value lna for reflectivity above $0 d B Z$, also supports the empirical relation for drizzle by (Sauvageot and Omar, 1987) as shown in table 1 where the lna is given as 5.77. The standard deviation of $\ln a$ is also high for profiles with maximum reflectivity above $-10 \mathrm{dBZ}$. The standard deviation of $\ln a$ is lowest between -30 to $-20 \mathrm{dBZ}$. The one-dimensional linear fit relating $\ln a$ and maximum radar reflectivity for clouds columns, is shown in black dashed line in figure 14 (b). As maximum reflectivity of the profile is showing better correlation with $l n a$ and the mean lna (red dashed line) coincides with linear fit. Therefore, the one dimensional linear equation relation between $\ln a$ and maximum reflectivity $\left(Z_{\max }\right)$ is given by
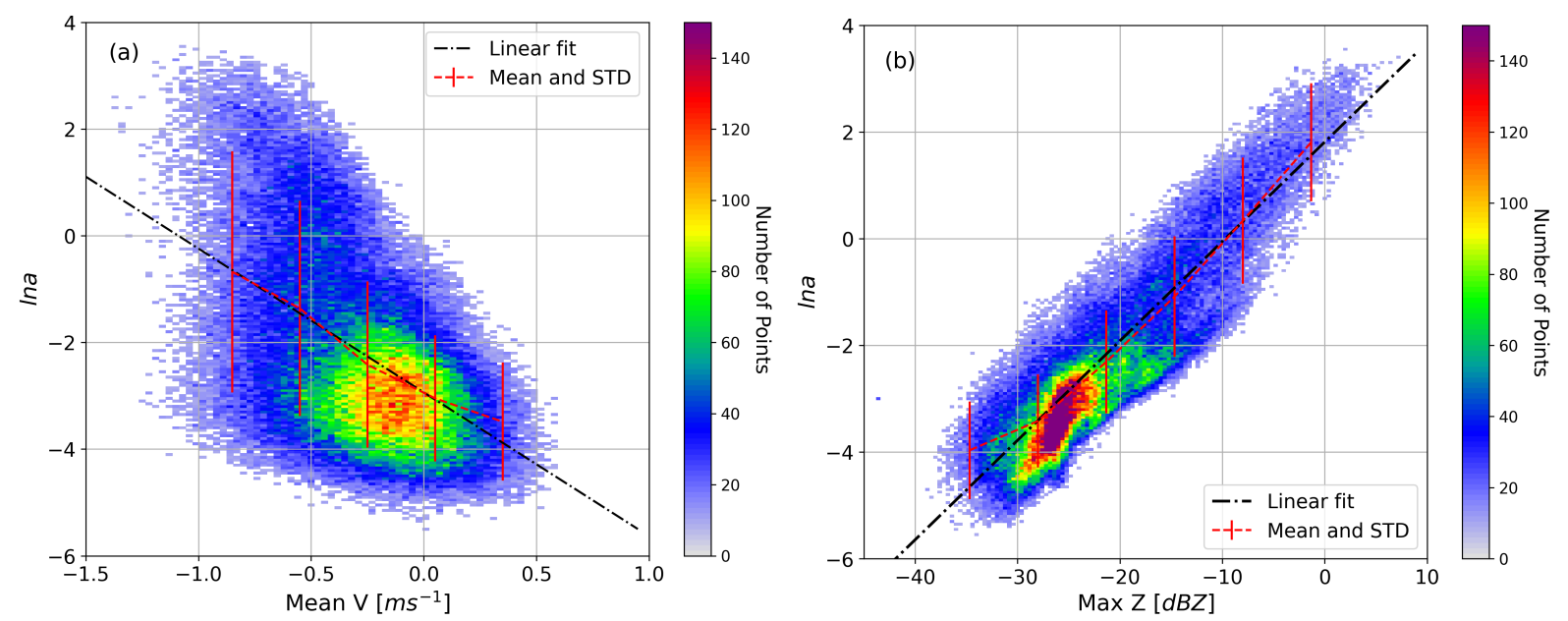

Figure 14. Correlation of (a) Mean velocity versus $\ln a$ and (b) Maximum reflectivity versus $\ln a$ for cloud profiles, where color bar indicates the number of profiles

$\ln a=0.186 \cdot Z_{\max }+1.829$

However, from an investigation by selecting 15 fog cases out of 39 cloud cases indicated that the coefficients of linear fit are slightly different for fog profile.

$\ln a=0.149 \cdot Z_{\max }+0.591$ 
To utilize the above relationships, it is necessary to differentiate between liquid cloud aloft and fog. This can be easily done by determining the cloud base height to identify fog, and hence specific climatology is applied to the profile.

\section{BASTA standalone LWC retrieval using climatology}

In this section, we describe the stand-alone approach and its assessment using MWR LWP retrieval as a reference. The climatological relation of $\ln a$ as a function of maximum radar reflectivity in the profile is used for the BASTA standalone retrieval when MWR observations are unavailable.

\subsection{BASTA standalone LWC retrieval approach}

The radar is not always accompanied by a MWR and therefore a solution must be proposed to improve the retrieval with knowledge of lna a priori. Since LWP information is not assimilated, thanks to the lna climatology for clouds and fog derived in section 6, this information can be used as lna a priori. lna for the profile can be linked to the maximum value of reflectivity detected in the profile using Eqs. (17) and (18) for clouds and fog respectively.

In this case, the observation vector ' $y$ ' contains only radar reflectivity of each cloud pixel, with $25 \%$ error in measurement, whereas the state vector still contains LWC and $\ln a$ both. Therefore, the Jacobian for a cloud profile with $n$ cloud pixels will have $n \times(n+1)$ elements. The variational method also allows us to control the contribution of $a$ priori information in the retrieval by providing error in a priori. A strong a priori of lna is required to constrain LWC retrieval therefore, low error in a priori of $\ln a$ is employed. In these standalone retrieval cases, $100 \%$ error in a priori of lna is used, because the standard deviation of lna in figure 14 is approximately 1 which is equivalent to $100 \%$ error in a priori. The climatology of $\ln a$ for fog from Eq. (18) is applied to the profile with cloud base less than $80 \mathrm{~m}$. Retrieval of LWC should be constrained by LWC a priori only to avoid non-physical values, therefore the error in a priori of LWC is taken $1000 \%$. In BASTA standalone retrieval setup, a priori of LWC is calculated using Atlas (1954) relation exactly the same as radar-MWR synergistic retrieval.

\subsection{BASTA standalone LWC retrieval first assessment using LWP retrieved from MWR}

With the details given above, LWC retrieval algorithm is adapted to utilize climatology of scaling factor with only radar reflectivity measurements from SIRTA. BASTA standalone retrieval algorithm is applied to the 39 selected cloud and fog cases from SIRTA.

Due to the absence of in-situ sensors at SIRTA for recording the distribution of the liquid water content in cloud and fog, the integrated LWP from the HATPRO microwave radiometer is utilized to assess the quality of the retrieved LWC for BASTA stand-alone retrieval. The retrieved LWP is calculated by vertically integrating the retrieved LWC. Because LWP information is not assimilated and strong lna a priori derived from climatology is constraining the retrieval, and hence lna is not a retrieved parameter. However, the variational framework allows lna to adjust around its climatology depending on radar reflectivity. In this case lna values falls within the range of known values from literature as shown in table 1. 


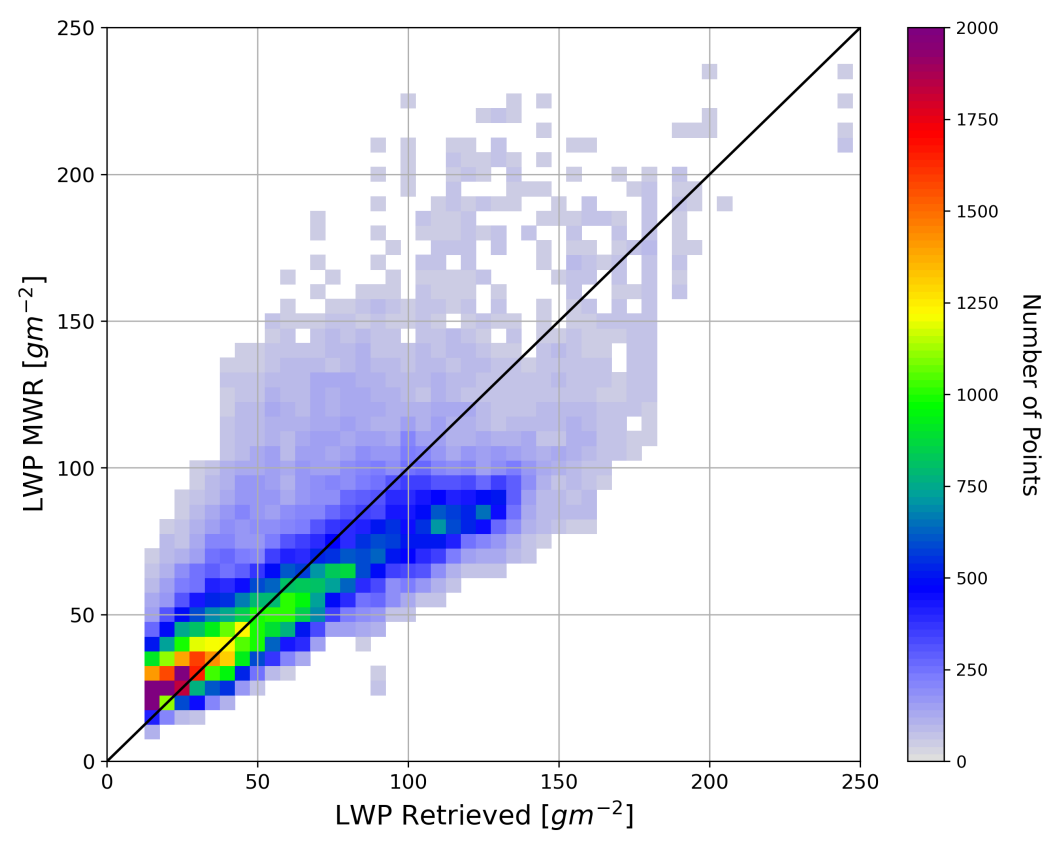

Figure 15. Comparison of retrieved LWP with LWP retrieved by HATPRO, where the black line represents the exact match of LWP for given profile.

Figure 15 presents the comparison of retrieved LWP from BASTA stand-alone retrieval with LWP retrieved from HATPRO microwave radiometer. Number density of profiles with LWP ranging from 10 to $250 \mathrm{gm}^{-2}$ are compared with LWP from BASTA stand-alone retrieval. Profile with retrieved LWP less than $50 \mathrm{gm}^{-2}$ shows good agreement with LWP from HATPRO. For the profile with higher LWP an increase in bias is clearly observed in figure 15, and the mean bias in LWP obtained as the difference between LWP from HATPRO and retrieved is $-21 \mathrm{gm}^{-2}$. The mean absolute percentage error in LWP w.r.t. LWP from HATPRO is $57.15 \%$. The relative error in LWP shown in figure 16 indicates that the majority of clouds has less than 35\% error in retrieved LWP. Because the climatology of the scaling factor constrains the retrieval, effective estimation of LWC can be made using only radar information when additional information is unavailable. By investigating the origin of biases, we discovered that the profiles with light drizzle droplets characteristics tend to overestimate the LWP by a large margin. The improvement in standalone retrieval can be made by classifying clouds with and without drizzle and using specific lna climatology for them.

\section{Summary and conclusions}

An algorithm for LWC estimation of warm clouds is proposed using a vertically pointing radar and microwave radiometer synergy. The algorithm also accounts for attenuation due to liquid cloud droplets. This algorithm is based on the hypothesis that LWC is related to reflectivity with a power-law fit, and one of the constants of the Z-LWC relationship is allowed to vary 


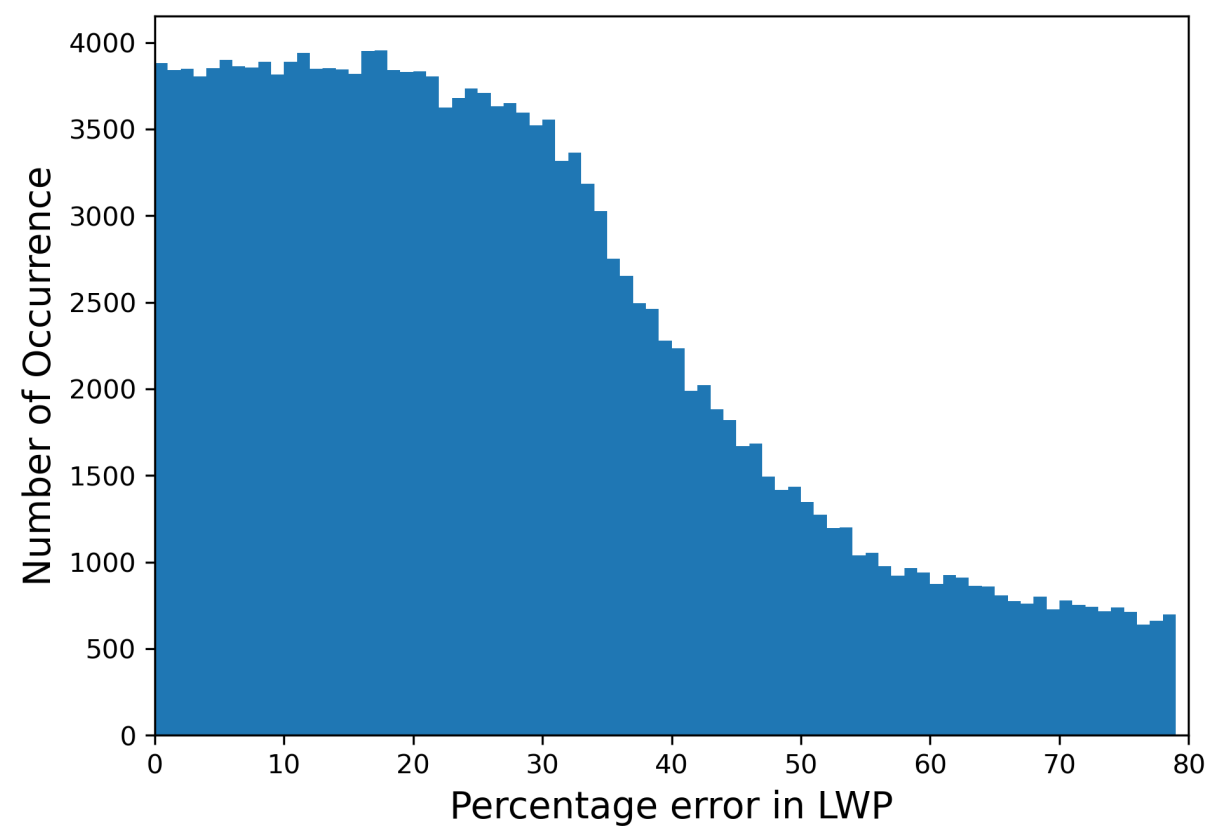

Figure 16. Percentage error in retrieved LWP with respect to LWP measured by MWR at SIRTA.

according to LWP retrieved by a MWR of the same cloud profile. The scaling factor of the relationship, lna is retrieved while the exponent $b$ is assumed constant. Therefore, the technique proposed in this study is equivalent to finding a suitable Z-LWC relationship consistent with the measured LWP for each cloud profile. This synergistic retrieval algorithm works seamlessly for liquid clouds and fog without prescribing the cloud type. The algorithm is implemented to a set of cloud and fog instances observed at SIRTA and the analysis is discussed in this paper. These retrievals have been used to develop a climatology of LWC and the scaling factor for warm clouds and fog. The application of derived climatology to estimate LWC for stand-alone radar observations is also presented in this paper. By utilizing the climatology of the scaling factor, this radar stand-alone method can provide continuous retrieval of LWC for warm clouds even in the absence of radiometer and other additional measurements. Although this climatology is developed using measurements from SIRTA observatory for limited cloud scenarios, a more extensive data collection from several measurement locations might be used to generate a more robust climatology of scaling factor.

Furthermore, the retrievals are compared against in-situ measurements for a fog and cloud case collected during the SOFOG3D field campaign. The comparison of LWC values estimated using this synergistic retrieval algorithm revealed that the fog and clouds were clearly distinct. The retrieved LWC was more consistent with stratus cloud than fog. A homogeneous cloud system is required for the comparison of retrieved LWC with in-situ measurements, or else the in-situ sensors must sample the same cloud or fog volume as radar. To assess the accuracy of algorithm for LWC estimates in various clouds types, in-situ measurements of several types of warm clouds like fog, low level stratus clouds with and without drizzle are required. 
However, drizzle in clouds is a substantial source of error in the retrieval. Because drizzle droplets are significantly larger than cloud droplets, power law may not be applicable in the Mie regime. As a result, the forward model exclusively for drizzle must incorporate Mie scattering or eventually another kind of relationship to link Z and LWC. A prospective work for such cloud columns is planned to separate drizzle and cloud pixels using Doppler velocity information and develop a forward model for drizzle. The variational framework discussed here can be modified to incorporate additional measurements such as Doppler velocity.

Another current limitation of our synergistic retrieval method is that it is applicable to profiles with LWP values greater than $10 \mathrm{gm}^{-2}$. A better a priori of lna can be proposed in future to estimate accurate LWC for low LWP profiles. Additionally, this retrieval method is not applicable when a mixed phase cloud overlap the liquid cloud layer, whereas ice cloud above the liquid cloud does not impact the LWC retrieval of the liquid layer.

As mentioned in the section 3.4, the radar reflectivity profiles can be contaminated by particles in the boundary layer. In the retrieval method, these airborne plankton must be categorized and hence not processed as hydrometeors. Ultimately, a sophisticated algorithm for the classification of hydrometeors to distinguish between fog, liquid cloud, and drizzle is required. The retrieval algorithm could be updated with an improved target classification scheme to apply two different scaling factors in one profile, especially when drizzle and cloud co-occur. Also, for multi-layered liquid clouds, different lna might be prescribed for each cloud layer with proper classification of hydrometeors. Improved classification of hydrometers for the BASTA standalone retrieval will improve the LWC retrieval because the range of scaling factor varies for different categories. Further, the climatology of scaling factor for different cloud types will improve the LWC retrieval.

We know that cloud LWC values can fluctuate both horizontally and vertically. The retrieval algorithm can be validated with in-situ measurements from aircraft, balloons, and UAVs flights with in-cloud sensors for diverse liquid clouds. Since UAVs and balloons travel at a slower speed than airplanes, which would allow them to sample the clouds more thoroughly. Note that some sites cannot be overflown by aircraft for safety reason. UAVs can be more efficient in terms of controlling them remotely, as the path of tethered balloons cannot be controlled. These platforms can, however, interfere with the radar signal. In order to avoid contaminating the radar signal, the samples must be taken from a volume that is close enough and least obstructing the radar. Before comparing the estimated values with in-situ data, it is necessary to verify if the cloud volume represented by radar and in-situ sample are the same. A well mixed or homogeneous cloud systems is ideal for validating such algorithms. Télédétection Atmosphérique, 2021) and https://sofog3d.aeris-data.fr/ respectively.

Code and data availability. The code developed in this study can be made publicly available upon request to the authors. 
https://doi.org/10.5194/amt-2022-3

Preprint. Discussion started: 31 January 2022

(C) Author(s) 2022. CC BY 4.0 License.

(c) (1)

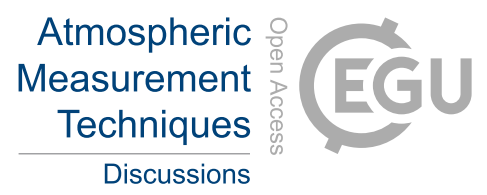

Author contributions. PV developed the retrieval algorithm and analyzed the results with support from JD. SJ organised and preprocessed the data used in this paper from SOFOG-3D. PM provided information and data from MWR, and FB provided the CDP data from SOFOG-

$7853 \mathrm{D}$ and relevant assistance. AB provided data from the AROME model used for sensitivity analysis. JCD provided the data from SIRTA. PV wrote the paper with contributions from all co-authors.

Competing interests. The authors declare that they have no conflict of interest.

Acknowledgements. This research has been funded by the French Association Nationale de la Recherche (ANRT) and the company Meteomodem. This research has been supported by the Université de Versailles Saint-Quentin-en-Yvelines (UVSQ), Université Paris-Saclay.

790 The authors would like to acknowledge SIRTA for providing the BASTA radar and MWR data used in this study. The authors would like to acknowledge the assistance team of SOFOG-3D campaign for the execution of the field experiments. 


\section{References}

Atlas, D.: THE ESTIMATION OF CLOUD PARAMETERS BY RADAR, Journal of the Atmospheric Sciences, 11, 309-317, https://doi.org/10.1175/1520-0469(1954)011<0309:TEOCPB>2.0.CO;2, publisher: American Meteorological Society Section: Journal of the Atmospheric Sciences, 1954.

Baedi, R. J. P., de Wit, J. J. M., Russchenberg, H. W. J., Erkelens, J. S., and Poiares Baptista, J. P. V.: Estimating effective radius and liquid water content from radar and lidar based on the CLARE98 data-set, Physics and Chemistry of the Earth, Part B: Hydrology, Oceans and Atmosphere, 25, 1057-1062, https://doi.org/10.1016/S1464-1909(00)00152-0, 2000.

Bell, A., Martinet, P., Caumont, O., Vié, B., Delanoë, J., Dupont, J.-C., and Borderies, M.: W-band radar observations for fog forecast improvement: an analysis of model and forward operator errors, Atmospheric Measurement Techniques, 14, 4929-4946, https://doi.org/10.5194/amt-14-4929-2021, 2021.

Bony, S. and Dufresne, J.-L.: Marine boundary layer clouds at the heart of tropical cloud feedback uncertainties in climate models, Geophysical Research Letters, 32, https://doi.org/10.1029/2005GL023851, _eprint: https://agupubs.onlinelibrary.wiley.com/doi/pdf/10.1029/2005GL023851, 2005.

Brousseau, P., Seity, Y., Ricard, D., and Léger, J.: Improvement of the forecast of convective activity from the AROME-France system, Quarterly Journal of the Royal Meteorological Society, 142, 2231-2243, https://doi.org/10.1002/qj.2822, 2016.

Cimini, D., Hewison, T. J., Martin, L., Güldner, J., Gaffard, C., and Marzano, F. S.: Temperature and humidity profile retrievals from groundbased microwave radiometers during TUC, Meteorologische Zeitschrift, 15, 45-56, 2006.

Crewell, S. and Löhnert, U.: Accuracy of cloud liquid water path from ground-based microwave radiometry 2. Sensor accuracy and synergy, Radio Science, 38, 7-1, 2003.

Delanoë, J. and Hogan, R. J.: A variational scheme for retrieving ice cloud properties from combined radar, lidar, and infrared radiometer, Journal of Geophysical Research: Atmospheres, 113, https://doi.org/10.1029/2007JD009000, _eprint: https://onlinelibrary.wiley.com/doi/pdf/10.1029/2007JD009000, 2008.

Delanoë, J., Protat, A., Vinson, J.-P., Brett, W., Caudoux, C., Bertrand, F., Chatelet, J. P. d., Hallali, R., Barthes, L., Haeffelin, M., and Dupont,

815 J.-C.: BASTA: A 95-GHz FMCW Doppler Radar for Cloud and Fog Studies, Journal of Atmospheric and Oceanic Technology, 33, 10231038, https://doi.org/10.1175/JTECH-D-15-0104.1, publisher: American Meteorological Society Section: Journal of Atmospheric and Oceanic Technology, 2016.

Dupont, J.-C., Haeffelin, M., Wærsted, E., Delanoe, J., Renard, J.-B., Preissler, J., and O’Dowd, C.: Evaluation of Fog and Low Stratus Cloud Microphysical Properties Derived from In Situ Sensor, Cloud Radar and SYRSOC Algorithm, Atmosphere, 9, 169, https://doi.org/10.3390/atmos9050169, number: 5 Publisher: Multidisciplinary Digital Publishing Institute, 2018.

Ellis, S. M. and Vivekanandan, J.: Liquid water content estimates using simultaneous S and $\mathrm{K}_{\text {a }}$ band radar measurements: DUALWAVELENGTH RADAR LWC ESTIMATES, Radio Science, 46, n/a-n/a, https://doi.org/10.1029/2010RS004361, 2011.

Fan, J., Wang, Y., Rosenfeld, D., and Liu, X.: Review of Aerosol-Cloud Interactions: Mechanisms, Significance, and Challenges, Journal of the Atmospheric Sciences, 73, 4221-4252, https://doi.org/10.1175/JAS-D-16-0037.1, publisher: American Meteorological Society Section: Journal of the Atmospheric Sciences, 2016.

Fox, N. I. and Illingworth, A. J.: The Retrieval of Stratocumulus Cloud Properties by Ground-Based Cloud Radar, Journal of Applied Meteorology and Climatology, 36, 485-492, https://doi.org/10.1175/1520-0450(1997)036<0485:TROSCP>2.0.CO;2, publisher: American Meteorological Society Section: Journal of Applied Meteorology and Climatology, 1997. 
Frisch, A. S., Fairall, C. W., and Snider, J. B.: Measurement of Stratus Cloud and Drizzle Parameters in ASTEX with a K $\alpha$-Band 0469(1995)052<2788:MOSCAD>2.0.CO;2, publisher: American Meteorological Society Section: Journal of the Atmospheric Sciences, 1995.

Frisch, A. S., Feingold, G., Fairall, C. W., Uttal, T., and Snider, J. B.: On cloud radar and microwave radiometer measurements of stratus cloud liquid water profiles, Journal of Geophysical Research: Atmospheres, 103, 23 195-23 197, https://doi.org/https://doi.org/10.1029/98JD01827,_eprint: https://agupubs.onlinelibrary.wiley.com/doi/pdf/10.1029/98JD01827, 1998.

Geerts, B. and Miao, Q.: The Use of Millimeter Doppler Radar Echoes to Estimate Vertical Air Velocities in the Fair-Weather Convective Boundary Layer, Journal of Atmospheric and Oceanic Technology, 22, 225-246, https://doi.org/10.1175/JTECH1699.1, publisher: American Meteorological Society Section: Journal of Atmospheric and Oceanic Technology, 2005.

Haeffelin, M., Barthès, L., Bock, O., Boitel, C., Bony, S., Bouniol, D., Chepfer, H., Chiriaco, M., Cuesta, J., Delanoë, J., Drobinski, P., Dufresne, J.-L., Flamant, C., Grall, M., Hodzic, A., Hourdin, F., Lapouge, F., Lemaître, Y., Mathieu, A., Morille, Y., Naud, C., Noël, V., O’Hirok, W., Pelon, J., Pietras, C., Protat, A., Romand, B., Scialom, G., and Vautard, R.: SIRTA, a ground-based atmospheric observatory for cloud and aerosol research, Annales Geophysicae, 23, 253-275, https://doi.org/10.5194/angeo-23-253-2005, publisher: Copernicus $\mathrm{GmbH}, 2005$.

Hartmann, D. L., Ockert-Bell, M. E., and Michelsen, M. L.: The Effect of Cloud Type on Earth's Energy Balance: Global Analysis, Journal of Climate, 5, 1281-1304, https://doi.org/10.1175/1520-0442(1992)005<1281:TEOCTO>2.0.CO;2, publisher: American Meteorological Society Section: Journal of Climate, 1992.

Hogan, R. J.: A Variational Scheme for Retrieving Rainfall Rate and Hail Reflectivity Fraction from Polarization Radar, Journal of Applied Meteorology and Climatology, 46, 1544-1564, https://doi.org/10.1175/JAM2550.1, publisher: American Meteorological Society Section: Journal of Applied Meteorology and Climatology, 2007.

Hogan, R. J., Gaussiat, N., and Illingworth, A. J.: Stratocumulus Liquid Water Content from Dual-Wavelength Radar, Journal of Atmospheric and Oceanic Technology, 22, 1207-1218, https://doi.org/10.1175/JTECH1768.1, 2005.

Illingworth, A. J., Hogan, R. J., O’Connor, E. J., Bouniol, D., Brooks, M. E., Delanoë, J., Donovan, D. P., Eastment, J. D., Gaussiat, N., Goddard, J. W. F., Haeffelin, M., Baltink, H. K., Krasnov, O. A., Pelon, J., and Tompkins, A. M.: Continuous Evaluation of Cloud Profiles in Seven Operational Models Using Ground-Based Observations, p. 16, 2007.

Krasnov, O. and Russchenberg, H.: A synergetic radar-lidar technique for the LWC retrieval in water clouds: Description and application to the Cloudnet data, 2005.

Lance, S., Brock, C. A., Rogers, D., and Gordon, J. A.: Water droplet calibration of the Cloud Droplet Probe (CDP) and inflight performance in liquid, ice and mixed-phase clouds during ARCPAC, Atmospheric Measurement Techniques, 3, 1683-1706, https://doi.org/10.5194/amt-3-1683-2010, publisher: Copernicus GmbH, 2010.

Lhermitte, R.: Attenuation and Scattering of Millimeter Wavelength Radiation by Clouds and Precipitation, Journal of Atmospheric and Oceanic Technology, 7, 464-479, https://doi.org/10.1175/1520-0426(1990)007<0464:AASOMW>2.0.CO;2, publisher: American Meteorological Society Section: Journal of Atmospheric and Oceanic Technology, 1990.

Liebe, H., Manabe, T., and Hufford, G.: Millimeter-wave attenuation and delay rates due to fog/cloud conditions, IEEE Transactions on Antennas and Propagation, 37, 1617-1612, https://doi.org/10.1109/8.45106, conference Name: IEEE Transactions on Antennas and Propagation, 1989. 
Löhnert, U., Crewell, S., Simmer, C., and Macke, A.: Profiling Cloud Liquid Water by Combining Active and Passive Microwave Measurements with Cloud Model Statistics, Journal of Atmospheric and Oceanic Technology, 18, 1354-1366, https://doi.org/10.1175/15200426(2001)018<1354:PCLWBC>2.0.CO;2, publisher: American Meteorological Society Section: Journal of Atmospheric and Oceanic Technology, 2001.

Maahn, M., Turner, D. D., Löhnert, U., Posselt, D. J., Ebell, K., Mace, G. G., and Comstock, J. M.: Optimal Estimation Retrievals and Their Uncertainties: What Every Atmospheric Scientist Should Know, Bulletin of the American Meteorological Society, 101, E1512-E1523, https://doi.org/10.1175/BAMS-D-19-0027.1, publisher: American Meteorological Society Section: Bulletin of the American Meteorological Society, 2020.

Maier, F., Bendix, J., and Thies, B.: Simulating Z-LWC Relations in Natural Fogs with Radiative Transfer Calculations for Future Application to a Cloud Radar Profiler, Pure and Applied Geophysics, 169, 793-807, https://doi.org/10.1007/s00024-011-0332-0, 2012.

Marke, T., Ebell, K., Löhnert, U., and Turner, D. D.: Statistical retrieval of thin liquid cloud microphysical properties using ground-based infrared and microwave observations, Journal of Geophysical Research: Atmospheres, 121, 14-558, 2016.

Martinet, P., Cimini, D., Burnet, F., Ménétrier, B., Michel, Y., and Unger, V.: Improvement of numerical weather prediction model analysis during fog conditions through the assimilation of ground-based microwave radiometer observations: a 1D-Var study, Atmospheric Measurement Techniques, 13, 6593-6611, 2020.

Miles, N. L., Verlinde, J., and Clothiaux, E. E.: Cloud droplet size distributions in low-level stratiform clouds, Journal of the Atmospheric Sciences, 57, 295-311, https://doi.org/10.1175/1520-0469(2000)057<0295:CDSDIL>2.0.CO;2, publisher: American Meteorological Society, 2000.

Ovtchinnikov, M. and Kogan, Y. L.: Evaluation of radar retrieval algorithms in stratiform clouds using large-eddy simulations, Journal of Geophysical Research: Atmospheres, 105, 17 351-17359, https://doi.org/https://doi.org/10.1029/2000JD900216, _eprint: https://agupubs.onlinelibrary.wiley.com/doi/pdf/10.1029/2000JD900216, 2000.

O’Connor, E. J., Hogan, R. J., and Illingworth, A. J.: Retrieving Stratocumulus Drizzle Parameters Using Doppler Radar and Lidar, Journal of Applied Meteorology and Climatology, 44, 14-27, https://doi.org/10.1175/JAM-2181.1, publisher: American Meteorological Society Section: Journal of Applied Meteorology and Climatology, 2005.

Rodgers, C. D.: Inverse Methods for Atmospheric Sounding: Theory and Practice, WORLD SCIENTIFIC, https://doi.org/10.1142/3171, 2000.

Rosenfeld, D., Sherwood, S., Wood, R., and Donner, L.: Climate Effects of Aerosol-Cloud Interactions, Science, 343, 379-380, https://doi.org/10.1126/science.1247490, 2014.

Sauvageot, H. and Omar, J.: Radar Reflectivity of Cumulus Clouds, Journal of Atmospheric and Oceanic Technology, 4, 264-272, https://doi.org/10.1175/1520-0426(1987)004<0264:RROCC>2.0.CO;2, publisher: American Meteorological Society Section: Journal of Atmospheric and Oceanic Technology, 1987.

Seity, Y., Brousseau, P., Malardel, S., Hello, G., Bénard, P., Bouttier, F., Lac, C., and Masson, V.: The AROME-France convective-scale operational model, Monthly Weather Review, 139, 976-991, 2011.

Stephens, G. L.: Cloud Feedbacks in the Climate System: A Critical Review, Journal of Climate, 18, 237-273, https://doi.org/10.1175/JCLI3243.1, publisher: American Meteorological Society Section: Journal of Climate, 2005.

Stephens, G. L., Vane, D. G., Boain, R. J., Mace, G. G., Sassen, K., Wang, Z., Illingworth, A. J., O’connor, E. J., Rossow, W. B., Durden, S. L., Miller, S. D., Austin, R. T., Benedetti, A., and Mitrescu, C.: THE CLOUDSAT MISSION AND THE A-TRAIN: A New Dimension of Space-Based Observations of Clouds and Precipitation, Bulletin of the American Meteorological Society, 83, 1771-1790, 
https://doi.org/10.1175/BAMS-83-12-1771, publisher: American Meteorological Society Section: Bulletin of the American Meteorological Society, 2002.

Toledo, F., Delanoë, J., Haeffelin, M., Dupont, J.-C., Jorquera, S., and Le Gac, C.: Absolute calibration method for frequencymodulated continuous wave (FMCW) cloud radars based on corner reflectors, Atmospheric Measurement Techniques, 13, 6853-6875, https://doi.org/10.5194/amt-13-6853-2020, publisher: Copernicus GmbH, 2020.

Vali, G. and Haimov, S.: Observed extinction by clouds at 95 GHz, IEEE Transactions on Geoscience and Remote Sensing, 39, 190-193, https://doi.org/10.1109/36.898682, conference Name: IEEE Transactions on Geoscience and Remote Sensing, 2001.

Vivekanandan, J., Ghate, V. P., Jensen, J. B., Ellis, S. M., and Schwartz, M. C.: A Technique for Estimating Liquid Droplet Diameter and Liquid Water Content in Stratocumulus Clouds Using Radar and Lidar Measurements, Journal of Atmospheric and Oceanic Technology, 37, 2145-2161, https://doi.org/10.1175/JTECH-D-19-0092.1, publisher: American Meteorological Society Section: Journal of Atmospheric and Oceanic Technology, 2020.

915 Wang, J. and Geerts, B.: Identifying drizzle within marine stratus with W-band radar reflectivity, Atmospheric research, 69, 1-27, publisher: Elsevier, 2003.

Ware, R., Solheim, F., Carpenter, R., Gueldner, J., Liljegren, J., Nehrkorn, T., and Vandenberghe, F.: Radiometric profiling of tropospheric temperature, humidity and cloud liquid, 2002.

Winker, D. M., Pelon, J., Coakley, J. A., Ackerman, S. A., Charlson, R. J., Colarco, P. R., Flamant, P., Fu, Q., Hoff, R. M., Kittaka, C., Kubar, T. L., Treut, H. L., Mccormick, M. P., Mégie, G., Poole, L., Powell, K., Trepte, C., Vaughan, M. A., and Wielicki, B. A.: The CALIPSO Mission: A Global 3D View of Aerosols and Clouds, Bulletin of the American Meteorological Society, 91, 1211-1230, https://doi.org/10.1175/2010BAMS3009.1, publisher: American Meteorological Society Section: Bulletin of the American Meteorological Society, 2010.

Wood, R., Mechoso, C. R., Bretherton, C. S., Weller, R. A., Huebert, B., Straneo, F., Albrecht, B. A., Coe, H., Allen, G., Vaughan, G., Daum, P., Fairall, C., Chand, D., Gallardo Klenner, L., Garreaud, R., Grados, C., Covert, D. S., Bates, T. S., Krejci, R., Russell, L. M., de Szoeke, S., Brewer, A., Yuter, S. E., Springston, S. R., Chaigneau, A., Toniazzo, T., Minnis, P., Palikonda, R., Abel, S. J., Brown, W. O. J., Williams, S., Fochesatto, J., Brioude, J., and Bower, K. N.: The VAMOS Ocean-Cloud-Atmosphere-Land Study Regional Experiment (VOCALSREx): goals, platforms, and field operations, Atmospheric Chemistry and Physics, 11, 627-654, https://doi.org/10.5194/acp-11-627-2011, 2011.

930 Wærsted, E. G., Haeffelin, M., Dupont, J.-C., Delanoë, J., and Dubuisson, P.: Radiation in fog: quantification of the impact on fog liquid water based on ground-based remote sensing, Atmospheric Chemistry and Physics, 17, 10 811-10 835, https://doi.org/10.5194/acp-1710811-2017, publisher: Copernicus GmbH, 2017.

Zhu, L., Suomalainen, J., Liu, J., Hyyppä, J., Kaartinen, H., and Haggren, H.: A Review: Remote Sensing Sensors, IntechOpen, https://doi.org/10.5772/intechopen.71049, publication Title: Multi-purposeful Application of Geospatial Data, 2017. 